\title{
Estimates for iterated commutators of multilinear square fucntions with Dini-type kernels
}

Zengyan $\mathrm{Si}^{1 *}$ and Qingying Xue $\mathrm{e}^{2,3}$

"Correspondence:

zengyan@hpu.edu.cn

'School of Mathematics and Information Science, Henan

Polytechnic University, Jiaozuo, People's Republic of China

Full list of author information is available at the end of the article

\begin{abstract}
Let $T_{\Pi \vec{b}}$ be the commutator generated by a multilinear square function and Lipschitz functions with kernel satisfying Dini-type condition. We show that $T_{\Pi \vec{b}}$ is bounded from product Lebesgue spaces into Lebesgue spaces, Lipschitz spaces, and Triebel-Lizorkin spaces.
\end{abstract}

Keywords: Multilinear square fucntions; Triebel-Lizorkin spaces; Lipschitz spaces; Iterated commutators

\section{Introduction}

Let $A(x)$ be an elliptic $n \times n$ matrix with complex-valued entries that are merely bounded and measurable, and let $T=\operatorname{div}(A(x) \nabla)$. The well-known problem of Kato is to show the boundedness of $T^{1 / 2}$ from the Sobolev space $H^{1}\left(\mathbb{R}^{n}\right)$ to $L^{2}\left(\mathbb{R}^{n}\right)$. Fabes et al. [6] studied a family of multilinear square functions and applied it to the Kato problem. In fact, they obtained a collection of multilinear Littlewood-Paley estimates and then applied them to two problems in partial differential equations. The first problem is the estimation of the square root of an elliptic operator in divergence form, and the second is the estimation of solutions to the Cauchy problem for nondivergence-form parabolic equations. Such a square function has important applications in PDEs and other fields' we refer to [1-7, 9, $10,13,14,17-19]$ and the references therein. We now give the definition of the multilinear square function of type $\omega(t)$.

Suppose that $\omega(t):[0, \infty) \mapsto[0, \infty)$ is a nondecreasing function with $0<\omega(1)<\infty$. For $a>0$, we say that $\omega \in \operatorname{Dini}(a)$ if

$$
|\omega|_{\operatorname{Dini}(a)}=\int_{0}^{1} \omega^{a}(t) \frac{d t}{t}<\infty .
$$

Let $K_{t}\left(x, y_{1}, \ldots, y_{m}\right)$ be a locally integrable function defined away from the diagonal $x=$ $y_{1}=\cdots=y_{m}$ in $\left(\mathbb{R}^{n}\right)^{m+1}$. We say that $K_{t}\left(x, y_{1}, \ldots, y_{m}\right)$ is a kernel of type $\omega(t)$ if there is a positive constant $A$ such that the following conditions hold.

Size condition:

$$
\left(\int_{0}^{\infty}\left|K_{t}\left(x, y_{1}, \ldots, y_{m}\right)\right|^{2} \frac{d t}{t}\right)^{\frac{1}{2}} \leq \frac{A}{\left(\sum_{j=1}^{m}\left|x-y_{j}\right|\right)^{m n}} .
$$

(0) The Author(s) 2018. This article is distributed under the terms of the Creative Commons Attribution 4.0 International License (http://creativecommons.org/licenses/by/4.0/), which permits unrestricted use, distribution, and reproduction in any medium, provided you give appropriate credit to the original author(s) and the source, provide a link to the Creative Commons license, and indicate if changes were made. 
Smoothness condition:

$$
\begin{aligned}
& \left(\int_{0}^{\infty}\left|K_{t}\left(z, y_{1}, \ldots, y_{m}\right)-K_{t}\left(x, y_{1}, \ldots, y_{m}\right)\right|^{2} \frac{d t}{t}\right)^{\frac{1}{2}} \\
& \quad \leq \frac{A}{\left(\sum_{j=1}^{m}\left|x-y_{j}\right|\right)^{m n}} \omega\left(\frac{|z-x|}{\sum_{j=1}^{m}\left|x-y_{j}\right|}\right)
\end{aligned}
$$

whenever $|z-x| \leq \frac{1}{2} \max _{j=1}^{m}\left|x-y_{j}\right|$, and

$$
\begin{aligned}
& \left(\int_{0}^{\infty}\left|K_{t}\left(x, y_{1}, \ldots, y_{j}, \ldots, y_{m}\right)-K_{t}\left(x, y_{1}, \ldots, y_{j}^{\prime}, \ldots, y_{m}\right)\right|^{2} \frac{d t}{t}\right)^{\frac{1}{2}} \\
& \quad \leq \frac{A}{\left(\sum_{j=1}^{m}\left|x-y_{j}\right|\right)^{m n}} \omega\left(\frac{\left|y_{j}-y_{j}^{\prime}\right|}{\sum_{j=1}^{m}\left|x-y_{j}\right|}\right)
\end{aligned}
$$

whenever $\left|y_{j}-y_{j}^{\prime}\right| \leq \frac{1}{2} \max _{j=1}^{m}\left|x-y_{j}\right|$.

For any $x \notin \bigcap_{j=1}^{m} \operatorname{supp} f_{j}$ and $f_{j} \in C_{c}^{\infty}\left(\mathbb{R}^{n}\right)$, we say $T$ is a multilinear square function of type $\omega(t)$ if

$$
T(\vec{f})(x)=\left(\int_{0}^{\infty}\left|\int_{\left(\mathbb{R}^{n}\right)^{m}} K_{t}\left(x, y_{1}, \ldots, y_{m}\right) \prod_{j=1}^{m} f_{j}\left(y_{j}\right) d y_{1} \cdots d y_{m}\right|^{2} \frac{d t}{t}\right)^{\frac{1}{2}} .
$$

In this paper, we always assume that $T$ can be extended to bounded operators from $L^{q_{1}} \times \cdots \times L^{q_{m}}$ to $L^{q}$ for some $1<q, q_{1}, \ldots, q_{m}<\infty$ with $\frac{1}{q_{1}}+\cdots+\frac{1}{q_{m}}=\frac{1}{q}$.

Remark 1.1 When $\omega(x)=x^{\gamma}$ for some $\gamma>0$, the boundedness of a multilinear square function was studied by Xue et al. [18].

Definition 1.2 (Iterated commutators) Given a collection of locally integrable functions $\vec{b}=\left(b_{1}, \ldots, b_{m}\right)$, the iterated commutator of a multilinear square function is defined by

$$
\begin{aligned}
& T_{\Pi \vec{b}}(\vec{f})(x) \\
& =\left(\int_{0}^{\infty} \mid \int_{\left(\mathbb{R}^{n}\right)^{m}} \prod_{j=1}^{m}\left(b_{j}(x)-b_{j}\left(y_{j}\right)\right) K\left(x, y_{1}, \ldots, y_{m}\right) K_{t}\left(x, y_{1}, \ldots, y_{m}\right)\right. \\
& \left.\quad \times\left.\prod_{j=1}^{m} f_{j}\left(y_{j}\right) d y_{1} \cdots d y_{m}\right|^{2} \frac{d t}{t}\right)^{\frac{1}{2}} .
\end{aligned}
$$

Definition 1.3 (Commutators in the $j$ th entry) Given a collection of locally integrable functions $\vec{b}=\left(b_{1}, \ldots, b_{m}\right)$, we define the commutator of a multilinear square function $T$ as

$$
[\vec{b}, T](\vec{f})=T_{\vec{b}}\left(f_{1}, \ldots, f_{m}\right)=\sum_{j=1}^{m} T_{\vec{b}}^{j}(\vec{f})
$$

where each term is the commutator of $b_{j}$, and $T$ in the jth entry of $T$, that is,

$$
T_{\vec{b}}^{j}(\vec{f})=b_{j} T\left(f_{1}, \ldots, f_{j}, \ldots, f_{m}\right)-T\left(f_{1}, \ldots, b_{j} f_{j}, \ldots, f_{m}\right) .
$$


For the commutators generated by the multilinear Calderón-Zygmud-type singular integrals and Lipschitz functions with the kernel of standard estimates, Wang and $\mathrm{Xu}$ [16] and $\mathrm{Mo}$ and $\mathrm{Lu}$ [11] obtained the boundedness from a product of Lebesgue spaces to the Lebesgue space, to the homogenous Triebel-Lizorkin space, and to Lipschitz spaces, respectively. Motivated by these results, we study the boundedness of commutators generated by the multilinear square functions and Lipschitz functions. The main results of this paper are as follows.

Theorem 1.1 Let $T$ be a multilinear square function of type $\omega(t)$ with $\omega \in \operatorname{Dini}(1)$. Suppose $b_{j} \in \dot{\lambda}_{\beta_{j}}$ with $0<\beta_{j}<1$ for $j=1, \ldots$, m and $\beta=\beta_{1}+\cdots+\beta_{m}$. If $1<p_{1}, \ldots, p_{m}<\infty, 0<q<\infty$, and $1 / p_{j}>\beta_{j} / n$ with $1 / q=1 / p_{1}+\cdots+1 / p_{m}-\beta / n$, then $T_{\Pi \vec{b}}$ can be extended to a bounded operator from $L^{p_{1}} \times \cdots \times L^{p_{m}}$ into $L^{q}$.

Theorem 1.2 Let $T$ be a multilinear square function of type $\omega(t)$ with $\omega \in \operatorname{Dini}(1)$. Suppose $b_{j} \in \dot{\lambda}_{\beta_{j}}$ with $0<\beta_{j}<1$ for $j=1, \ldots$, m and $\beta=\beta_{1}+\cdots+\beta_{m}$. Let $1<p_{1}, \ldots, p_{m}<\infty, 0<$ $1 / p_{j}<\beta_{j} / n$, and $0<\beta-n / p<1$ with $1 / p=1 / p_{1}+\cdots+1 / p_{m}$. If $\omega$ satisfies

$$
\int_{0}^{1} \frac{\omega(t)}{t^{1+\beta-n / p}} d t<\infty
$$

then $T_{\Pi \vec{b}}$ can be extended to a bounded operator from $L^{p_{1}} \times \cdots \times L^{p_{m}}$ into Lipschitz space $\dot{\lambda}_{\beta-n / p}$.

Theorem 1.3 Let $T$ be a multilinear square function of type $\omega(t)$ with $\omega \in \operatorname{Dini}(1)$. Suppose $b_{j} \in \dot{\lambda}_{\beta_{j}}$ with $0<\beta_{j}<1$ for $j=1, \ldots, m$ and $\beta=\beta_{1}+\cdots+\beta_{m}$. If $1<p_{1}, \ldots, p_{m}<\infty$ with $1 / p=1 / p_{1}+\cdots+1 / p_{m}$ and $\omega$ satisfies

$$
\int_{0}^{1} \frac{\omega(t)}{t^{1+\beta}} d t<\infty
$$

then $T_{\Pi \vec{b}}$ can be extended to a bounded operator from $L^{p_{1}} \times \cdots \times L^{p_{m}}$ into the TriebelLizorkin space $\dot{F}_{p}^{\beta, \infty}$.

The paper is organized as follows. Some definitions and preliminaries are given in Sect. 2. In Sect. 3, we focus ourselves on a key lemma, which will be used in the proof of Theorem 1.1. The proofs of Theorems 1.2 and 1.3 are given in Sect. 4 .

\section{Preliminaries}

Definition 2.1 For $\delta>0, M_{\delta}$ is the maximal function defined by

$$
M_{\delta} f(x)=M\left(|f|^{\delta}\right)^{\frac{1}{\delta}}(x)=\left(\sup _{Q \ni x} \frac{1}{|Q|} \int_{Q}|f(y)|^{\delta} d y\right)^{\frac{1}{\delta}} .
$$

In addition, $M^{\sharp}$ is the sharp maximal function of Feffeman and Stein,

$$
M^{\sharp} f(x)=\sup _{Q \ni x} \inf _{c} \frac{1}{|Q|} \int_{Q}|f(y)-c| d y \approx \sup _{B \ni x} \frac{1}{|Q|} \int_{Q}\left|f(y)-f_{Q}\right| d y,
$$


and

$$
M_{\delta}^{\sharp} f(x)=M^{\sharp}\left(|f|^{\delta}\right)^{\frac{1}{\delta}}(x) .
$$

Given a locally integrable function $f$, for $0 \leq \beta<n$, we define the fractional maximal function

$$
M_{r, \beta} f(x)=\sup _{x \in B}\left(\frac{1}{|B|^{1-\beta r / n}} \int_{B}|f(y)|^{r} d y\right)^{\frac{1}{r}}, \quad r \geq 1 .
$$

If $\beta=0$ and $r=1$, then $M_{0,1} f=M f$ denotes the usual Hardy-Littlewood maximal function. When $\beta=0$, we denote $M_{r, \beta}$ simply by $M_{r}$.

Chanillo [1] proved that if $0<\beta<n, 0<r<p<n / \beta$, and $1 / q=1 / p-\beta / n$, then

$$
\left\|M_{r, \beta}\right\|_{q} \leq C\|f\|_{p}
$$

Definition 2.2 ([12]) For $\beta>0$, the homogenous Lipschitz space $\dot{\lambda}_{\beta}\left(\mathbb{R}^{n}\right)$ is the space of functions $f$ such that

$$
\|f\|_{i_{\beta}\left(\mathbb{R}^{n}\right)}=\sup _{x, h \in \mathbb{R}^{n}, h \neq 0} \frac{\left|\Delta_{h}^{[\beta]+1} f(x)\right|}{|h|^{\beta}}<\infty
$$

where $\Delta_{h}^{k}$ denotes the $k$ th difference operator.

To prove our theorem, we need the following lemmas.

Lemma 2.1 ([12]) Let $b \in \dot{\lambda}_{\beta}, 0<\beta<1$. For any cubes $Q^{\prime}, Q$ in $\mathbb{R}^{n}$ such that $Q^{\prime} \subset Q$, we have

$$
\left|b_{Q^{\prime}}-b_{Q}\right| \leq C\|b\|_{\dot{\lambda}_{\beta}}|Q|^{\beta / n}
$$

Lemma $2.2([12])$

(1) For $0<\beta<1$ and $1 \leq q<\infty$, we have

$$
\|f\|_{\lambda_{\beta}} \approx \sup _{Q} \frac{1}{|Q|^{1+n / \beta}} \int_{Q}\left|f-f_{Q}\right| \approx \sup _{Q} \frac{1}{|Q|^{n / \beta}}\left(\int_{Q}\left|f-f_{Q}\right|^{q}\right)^{\frac{1}{q}} .
$$

(2) For $0<\beta<1$ and $1 \leq p<\infty$, we have

$$
\|f\|_{\dot{F}_{p}^{\beta, \infty}} \approx\left\|\sup _{Q} \frac{1}{|Q|^{1+n / \beta}} \int_{Q}\left|f-f_{Q}\right|\right\|_{L^{p}} .
$$

Lemma 2.3 ([15]) Let $\frac{1}{p}=\frac{1}{p_{1}}+\cdots+\frac{1}{p_{m}}$ and $\vec{\omega} \in A_{\vec{p}}$. Let $T$ be a multilinear square function of type $\omega(t)$ with $\omega \in \operatorname{Dini}(1)$.

(1) If $1<p_{1}, \ldots, p_{m}<\infty$, then

$$
\|T \vec{f}\|_{L^{p}\left(v_{\vec{\omega}}\right)} \leq C \prod_{i=1}^{m}\left\|f_{i}\right\|_{L^{p_{i}\left(\omega_{i}\right)}} .
$$


(2) If $1 \leq p_{1}, \ldots, p_{m}<\infty$, then

$$
\|T \vec{f}\|_{L^{p, \infty}\left(v_{\vec{\omega}}\right)} \leq C \prod_{i=1}^{m}\left\|f_{i}\right\|_{L^{p_{i}}\left(\omega_{i}\right)} .
$$

\section{Proof of Theorem 1.1}

To prove Theorem 1.1, we need the following estimates for $T_{\Pi \vec{b}}$ and $T_{\vec{b}}^{j}$. We just consider the case $m=2$ for simplicity; our method still holds for general $m$ with little modifications.

Lemma 3.1 Let $0<\delta<\epsilon<1 / 2$, and let $T$ be a bilinear square function of type $\omega(t)$ with $\omega \in \operatorname{Dini}(1)$.

(i) If $b_{1} \in \dot{\wedge}_{\beta_{1}}$ and $b_{2} \in \dot{\Lambda}_{\beta_{2}}$ with $0<\beta_{1}, \beta_{2}<1$ such that $\beta_{1}+\beta_{2}=\beta$, then

$$
\begin{aligned}
M_{\delta}^{\sharp} T_{\Pi \vec{b}}\left(f_{1}, f_{2}\right)(x) \leq & C\left\{\prod_{i=1}^{2}\left\|b_{i}\right\|_{{\dot{\beta}}_{i}} M_{\epsilon, \beta}\left(T\left(f_{1}, f_{2}\right)\right)(x)\right. \\
& +\left\|b_{1}\right\|_{{\dot{\beta_{1}}}_{1}} M_{\epsilon, \beta_{1}}\left(T_{\vec{b}}^{2}\left(f_{1}, f_{2}\right)\right)(x) \\
& +\left\|b_{2}\right\|_{{\dot{\beta}}_{1}} M_{\epsilon, \beta_{2}}\left(T_{\vec{b}}^{1}\left(f_{1}, f_{2}\right)\right)(x) \\
& \left.+\prod_{i=1}^{2}\left\|b_{i}\right\|_{{\dot{\beta_{i}}}_{i}} M_{1, \beta_{1}}\left(f_{1}\right)(x) M_{1, \beta_{2}}\left(f_{2}\right)(x)\right\} .
\end{aligned}
$$

(ii) If $b_{j} \in \dot{\wedge}_{\beta}, j=1,2$, and $0<\beta<1$, then

$$
M_{\delta}^{\sharp} T_{\vec{b}}^{j}\left(f_{1}, f_{2}\right)(x) \leq C\left\|b_{j}\right\|_{\dot{\lambda}_{\beta}}\left\{M_{\epsilon, \beta}\left(T\left(f_{1}, f_{2}\right)\right)(x)+M_{1, \beta}\left(f_{j}\right)(x) M\left(f_{k}\right)(x)\right\},
$$

where $k \neq j, k=1,2$.

Proof Fix a point $x$ and a cube $Q\left(x_{Q}, l\right)$ containing $x$ with side-length $l$, and set $Q^{*}=$ $8 \sqrt{n} Q=Q\left(x_{Q}, 8 \sqrt{n} l\right)$. We split $f_{j}$ as $f_{j}=f_{j}^{0}+f_{j}^{\infty}$, where $f_{j}^{0}=f_{j} \chi_{Q^{*}}$ and $f_{j}^{\infty}=f_{j} \chi_{\mathbb{R}^{n} \backslash Q^{*}}$ for $j=1,2$. As is well known, to obtain (3.1), it suffices to show that

$$
\begin{aligned}
& \left(\frac{1}{|Q|} \int_{Q}\left|T_{\Pi \vec{b}}\left(f_{1}, f_{2}\right)(z)-c\right|^{\delta} d z\right)^{\frac{1}{\delta}} \\
& \quad \leq C\left\{\prod_{i=1}^{2}\left\|b_{i}\right\|_{{\dot{\beta_{i}}}_{i}} M_{\epsilon, \beta}\left(T\left(f_{1}, f_{2}\right)\right)(x)+\left\|b_{1}\right\|_{{\dot{\beta_{1}}}_{1}} M_{\epsilon, \beta_{1}}\left(T_{\vec{b}}^{2}\left(f_{1}, f_{2}\right)\right)(x)\right. \\
& \left.\quad+\left\|b_{2}\right\|_{{\dot{\beta_{1}}}_{1}} M_{\epsilon, \beta_{2}}\left(T_{\vec{b}}^{1}\left(f_{1}, f_{2}\right)\right)(x)+\prod_{i=1}^{2}\left\|b_{i}\right\|_{{\dot{\beta_{i}}}_{i}} M_{1, \beta_{1}}\left(f_{1}\right)(x) M_{1, \beta_{2}}\left(f_{2}\right)(x)\right\}
\end{aligned}
$$

for some constant $c$ to be determined.

Let $\lambda_{1}=\left(b_{1}\right)_{Q^{*}}$ and $\lambda_{2}=\left(b_{2}\right)_{Q^{*}}$. The sublinearity of $T_{\Pi \vec{b}}$ leads to

$$
\begin{aligned}
& \left|T_{\Pi \vec{b}}\left(f_{1}, f_{2}\right)(z)-c\right| \\
& \quad \leq\left|\left(b_{1}(z)-\lambda_{1}\right)\left(b_{2}(z)-\lambda_{2}\right) T\left(f_{1}, f_{2}\right)(z)\right|+\left|\left(b_{1}(z)-\lambda_{1}\right) T_{\vec{b}}^{2}\left(f_{1}, f_{2}\right)(z)\right| \\
& \quad+\left|\left(b_{2}(z)-\lambda_{2}\right) T_{\vec{b}}^{1}\left(f_{1}, f_{2}\right)(z)\right|+\left|T\left(\left(b_{1}-\lambda_{1}\right) f_{1},\left(b_{2}-\lambda_{2}\right) f_{2}\right)(z)-c\right| .
\end{aligned}
$$


Thus we have

$$
\begin{aligned}
\left(\frac{1}{|Q|} \int_{Q}\left|T_{\Pi \vec{b}}\left(f_{1}, f_{2}\right)(z)-c\right|^{\delta} d z\right)^{\frac{1}{\delta}} & \\
\leq & \left(\frac{1}{|B|} \int_{Q}\left|\left(b_{1}(z)-\lambda_{1}\right)\left(b_{2}(z)-\lambda_{2}\right) T\left(f_{1}, f_{2}\right)(z)\right|^{\delta} d z\right)^{\frac{1}{\delta}} \\
& +\left(\frac{1}{|Q|} \int_{Q}\left|\left(b_{1}(z)-\lambda_{1}\right) T_{\vec{b}}^{2}\left(f_{1}, f_{2}\right)(z)\right|^{\delta} d z\right)^{\frac{1}{\delta}} \\
& +\left(\frac{1}{|Q|} \int_{Q}\left|\left(b_{2}(z)-\lambda_{2}\right) T_{\vec{b}}^{1}\left(f_{1}, f_{2}\right)(z)\right|^{\delta} d z\right)^{\frac{1}{\delta}} \\
& +\left(\frac{1}{|Q|} \int_{Q}\left|T\left(\left(b_{1}-\lambda_{1}\right) f_{1},\left(b_{2}-\lambda_{2}\right) f_{2}\right)(z)-c\right|^{\delta} d z\right)^{\frac{1}{\delta}} \\
\doteq & T_{1}+T_{2}+T_{3}+T_{4} .
\end{aligned}
$$

We now observe the elementary inequality

$$
\left|b(z)-b_{Q}\right| \leq C|Q|^{\beta / n}\|b\|_{\dot{\lambda}_{\beta}}
$$

which follows from the fact $z \in Q$ and $b \in \dot{\lambda}_{\beta}$. From Hölder's inequality and the assumption $\beta_{1}+\beta_{2}=\beta$, for $0<\delta<\epsilon<1 / 2$, we have

$$
\begin{aligned}
T_{1} & \leq \prod_{i=1}^{2}\left\|b_{i}\right\|_{{\dot{\beta}}_{i}}\left(\frac{1}{|Q|^{1-\frac{\delta \beta}{n}}} \int_{Q}\left|T\left(f_{1}, f_{2}\right)(z)\right|^{\delta} d z\right)^{\frac{1}{\delta}} \\
& \leq \prod_{i=1}^{2}\left\|b_{i}\right\|_{{\dot{\lambda}}_{i}}\left(\frac{1}{|Q|^{1-\frac{\epsilon \beta}{n}}} \int_{Q}\left|T\left(f_{1}, f_{2}\right)(z)\right|^{\epsilon} d z\right)^{\frac{1}{\epsilon}} \\
& \leq C \prod_{i=1}^{2}\left\|b_{i}\right\|_{{\dot{\beta_{\beta}}}_{i}} M_{\epsilon, \beta}\left(T\left(f_{1}, f_{2}\right)\right)(x) .
\end{aligned}
$$

Similarly, we have

$$
T_{2} \leq C\left\|b_{1}\right\|_{{\dot{\beta_{1}}}_{1}} M_{\epsilon, \beta_{1}}\left(T_{\vec{b}}^{2}\left(f_{1}, f_{2}\right)\right)(x)
$$

and

$$
T_{3} \leq\left\|b_{2}\right\|_{{\dot{\beta_{2}}}_{2}} M_{\epsilon, \beta_{2}}\left(T_{\vec{b}}^{1}\left(f_{1}, f_{2}\right)\right)(x) .
$$

Now we deal with $T_{4}$. Set $c=T\left(\left(b_{1}-\lambda_{1}\right) f_{1}^{\infty},\left(b_{2}-\lambda_{2}\right) f_{2}^{\infty}\right)(x)$. We may bound $T_{4}$ as

$$
T_{4} \leq T_{41}+T_{42}+T_{43}+T_{44}
$$

where

$$
T_{41}=\left(\frac{1}{|Q|} \int_{Q}\left|T\left(\left(b_{1}-\lambda_{1}\right) f_{1}^{0},\left(b_{2}-\lambda_{2}\right) f_{2}^{0}\right)(z)\right|^{\delta} d x\right)^{\frac{1}{\delta}},
$$




$$
\begin{aligned}
& T_{42}=\left(\frac{1}{|Q|} \int_{Q}\left|T\left(\left(b_{1}-\lambda_{1}\right) f_{1}^{\infty},\left(b_{2}-\lambda_{2}\right) f_{2}^{0}\right)(z)\right|^{\delta} d z\right)^{\frac{1}{\delta}}, \\
& T_{43}=\left(\frac{1}{|Q|} \int_{Q}\left|T\left(\left(b_{1}-\lambda_{1}\right) f_{1}^{0},\left(b_{2}-\lambda_{2}\right) f_{2}^{\infty}\right)(z)\right|^{\delta} d z\right)^{\frac{1}{\delta}},
\end{aligned}
$$

and

$$
T_{44}=\left(\frac{1}{|Q|} \int_{Q}\left|T\left(\left(b_{1}-\lambda_{1}\right) f_{1}^{\infty},\left(b_{2}-\lambda_{2}\right) f_{2}^{\infty}\right)(z)-T\left(\left(b_{1}-\lambda_{1}\right) f_{1}^{\infty},\left(b_{2}-\lambda_{2}\right) f_{2}^{\infty}\right)(x)\right|^{\delta} d z\right)^{\frac{1}{\delta}} .
$$

For $T_{41}$, by Kolmogorov's inequality and Lemma 2.3 we get

$$
\begin{aligned}
T_{41} & \leq C\left\|T\left(\left(b_{1}-\lambda_{1}\right) f_{1}^{0},\left(b_{2}-\lambda_{2}\right) f_{2}^{0}\right)\right\|_{L^{1 / 2, \infty}\left(B, \frac{d x}{|Q|}\right)} \\
& \leq \frac{C}{|Q|} \int_{Q}\left|\left(b_{1}-\lambda_{1}\right) f_{1}^{0}(z)\right| d z \frac{1}{|Q|} \int_{Q}\left|\left(b_{2}-\lambda_{2}\right) f_{2}^{0}(z)\right| d z \\
& \leq C\left\|b_{1}\right\|{\dot{\lambda_{\beta_{1}}}}_{\|}\left\|b_{2}\right\|_{{\dot{\beta_{2}}}_{2}} M_{1, \beta_{1}}\left(f_{1}\right)(x) M_{1, \beta_{2}}\left(f_{2}\right)(x) .
\end{aligned}
$$

For any $y \in \mathbb{R}^{n} \backslash Q^{*}$ and $b \in \dot{\lambda}_{\beta}$, there exists $Q^{\prime}$ such that $Q^{*} \subset Q^{\prime}$ and $\left|y-x_{Q}\right| \sim\left|Q^{\prime}\right|^{1 / n}$. Then, from Lemma 2.1 we have

$$
\left|b(y)-b_{Q^{*}}\right| \leq\left|b(y)-b_{Q^{\prime}}\right|+\left|b_{Q^{\prime}}-b_{Q^{*}}\right| \leq C\|b\|_{\lambda_{\beta}}\left|y-x_{Q}\right|^{\beta} .
$$

For any $y_{2} \in\left(Q^{*}\right)^{c}$ and $z \in Q$, we have $\left|z-y_{2}\right| \sim\left|y_{2}-x_{Q}\right|$. By Minkowski's inequality and the size condition (1.1) we get

$$
\begin{aligned}
T_{43} \leq & \left(\frac{1}{|Q|} \int_{Q}\left|T\left(\left(b_{1}-\lambda_{1}\right) f_{1}^{0},\left(b_{2}-\lambda_{2}\right) f_{2}^{\infty}\right)(z)\right|^{\delta} d z\right)^{\frac{1}{\delta}} \\
\leq & C\left(\frac{1}{|Q|} \int_{Q} \mid \int_{\mathbb{R}^{n m}}\left(\int_{0}^{\infty}\left|K_{t}\left(z, y_{1}, y_{2}\right)\right|^{2} \frac{d t}{t}\right)^{1 / 2}\right. \\
& \left.\times\left.\left|\left(b_{1}\left(y_{1}\right)-\lambda_{1}\right) f_{1}^{0}\left(y_{1}\right)\right|\left|\left(b_{2}\left(y_{2}\right)-\lambda_{2}\right) f_{2}^{\infty}\left(y_{2}\right)\right| d y_{1} d y_{2}\right|^{\delta} d z\right)^{\frac{1}{\delta}} \\
\leq & C\left(\frac{1}{|Q|} \int_{Q}\left|\int_{Q^{*}} \int_{\mathbb{R}^{n} \backslash Q^{*}} \frac{\left|\left(b_{1}\left(y_{1}\right)-\lambda_{1}\right) f_{1}^{0}\left(y_{1}\right)\right|\left|\left(b_{2}\left(y_{2}\right)-\lambda_{2}\right) f_{2}^{\infty}\left(y_{2}\right)\right| d y_{1} d y_{2}}{\left(\left|z-y_{1}\right|+\left|z-y_{2}\right|\right)^{2 n}}\right|^{\delta} d z\right)^{\frac{1}{\delta}} \\
\leq & C\left\|b_{1}\right\|_{i_{\beta_{1}}} M_{1, \beta_{1}}\left(f_{1}\right)(x)|Q| \sum_{k=1}^{\infty} \int_{2^{k+3} \sqrt{n} Q \backslash 2^{k+2} \sqrt{n} Q} \frac{\left|f_{2}\left(y_{2}\right)\left(b_{2}\left(y_{2}\right)-\lambda_{2}\right)\right| d y_{2}}{\left|y_{2}-x_{Q}\right|^{2 n}} \\
\leq & C\left\|b_{1}\right\|_{i_{\beta_{1}}} M_{1, \beta_{1}}\left(f_{1}\right)(x)|Q| \sum_{k=1}^{\infty} \frac{1}{\left|2^{k+3} \sqrt{n} Q\right|^{1-\beta_{2}}} \int_{2^{k+3} \sqrt{n} Q}^{\left|f_{2}\left(y_{2}\right)\right| d y_{2}} \\
\leq & C\left\|b_{1}\right\|_{i_{\beta_{1}}}\left\|b_{2}\right\|_{\dot{\lambda}_{2}} M_{1, \beta_{1}}\left(f_{1}\right)(x) M_{1, \beta_{2}}\left(f_{2}\right)(x) \sum_{k=1}^{\infty} 2^{-k} \\
\leq & C\left\|b_{1}\right\|_{\dot{\lambda}_{\beta_{1}}}\left\|b_{2}\right\|_{\dot{\lambda}_{\beta_{2}}} M_{1, \beta_{1}}\left(f_{1}\right)(x) M_{1, \beta_{2}}\left(f_{2}\right)(x) .
\end{aligned}
$$

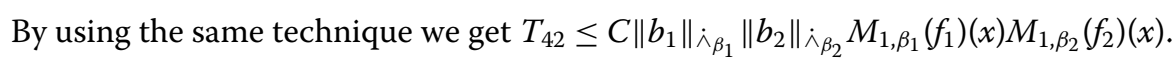


To estimate $T_{44}$, we use Minkowski's inequality and (1.2) and (3.3). Since $\left(\mathbb{R}^{n} \backslash Q^{*}\right)^{2} \subseteq$ $\mathbb{R}^{2 n} \backslash\left(Q^{*}\right)^{2} \subseteq \bigcup_{k=1}^{\infty}\left(2^{k+3} \sqrt{n} Q\right)^{2} \backslash\left(2^{k+2} \sqrt{n} Q\right)^{2}$, we deduce that

$$
\begin{aligned}
T_{44} \leq & \left(\frac{1}{|Q|} \int_{Q} \mid \int_{\mathbb{R}^{n m}}\left(\int_{0}^{\infty}\left|K_{t}(z, \vec{y})-K_{t}(x, \vec{y})\right|^{2} \frac{d t}{t}\right)^{1 / 2}\right. \\
& \left.\times\left.\prod_{i=1}^{2}\left|\left(b_{i}\left(y_{i}\right)-\lambda_{i}\right) f_{i}^{\infty}\left(y_{i}\right)\right| d \vec{y}\right|^{\delta} d z\right)^{\frac{1}{\delta}} \\
\leq & C\left(\frac{1}{|Q|} \int_{Q} \mid \int_{\left(\mathbb{R}^{n} \backslash Q^{*}\right)^{2}} \frac{1}{\left(\left|x-y_{1}\right|+\left|x-y_{2}\right|\right)^{2 n}} \omega\left(\frac{\left|z-x_{Q}\right|}{\left|x-y_{1}\right|+\left|x-y_{2}\right|}\right)\right. \\
& \left.\times\left.\prod_{i=1}^{2}\left|\left(b_{i}\left(y_{i}\right)-\lambda_{i}\right) f_{i}^{\infty}\left(y_{i}\right)\right| d \vec{y}\right|^{\delta} d z\right)^{\frac{1}{\delta}} \\
\leq & C \frac{1}{|Q|} \int_{Q} \sum_{k=1}^{\infty} \int_{\left(2^{k+3} \sqrt{n} Q \backslash 2^{k+2} \sqrt{n} Q\right)^{2}} \frac{1}{\left(\left|2^{k+3} \sqrt{n} Q\right|\right)^{2}} \omega\left(2^{-k}\right) \\
& \times \prod_{i=1}^{2}\left|\left(b_{i}\left(y_{i}\right)-\lambda_{i}\right) f_{i}^{\infty}\left(y_{i}\right)\right| d \vec{y} d z \\
\leq & C \sum_{k=1}^{\infty} \frac{1}{\left(\left|2^{k+3} \sqrt{n} Q\right|\right)^{2-\beta_{1}-\beta_{2}}} \int_{\left(2^{k+3} \sqrt{n} Q \backslash 2^{k+2} \sqrt{n} Q\right)^{2}} \omega\left(2^{-k}\right) \prod_{i=1}^{2}\left|f_{i}^{\infty}\left(y_{i}\right)\right| d \vec{y} \\
\leq & C\left\|b_{1}\right\| \dot{\lambda}_{\beta_{1}}\left\|b_{2}\right\| \dot{\lambda}_{\beta_{2}} M_{1, \beta_{1}}\left(f_{1}\right)(x) M_{1, \beta_{2}}\left(f_{2}\right)(x) .
\end{aligned}
$$

Combing all our estimates together, we obtain (3.1).

Now we are in the position to prove (3.2). It is sufficient to consider the operator with only one symbol. Fix $b \in \dot{\lambda}_{\beta}$ and consider the operator

$$
T_{b}(\vec{f})(x)=\left|b(x) T\left(f_{1}, f_{2}\right)(x)-T\left(b f_{1}, f_{2}\right)(x)\right| .
$$

We have to prove that

$$
M_{\delta}^{\sharp} T_{b}\left(f_{1}, f_{2}\right)(x) \leq C\|b\|_{\dot{\lambda}_{\beta}}\left\{M_{\epsilon, \beta}\left(T\left(f_{1}, f_{2}\right)\right)(x)+M_{1, \beta}\left(f_{1}\right)(x) M\left(f_{2}\right)(x)\right\} .
$$

Let $\lambda=b_{Q^{*}}$. We can control $T_{b}(\vec{f})(x)$ as

$$
T_{b}(\vec{f})(x) \leq|(b(x)-\lambda)| T\left(f_{1}, \ldots, f_{m}\right)(x)+T\left((b-\lambda) f_{1}, \ldots, f_{m}\right)(x) .
$$

Then, for any constant $c$, we obtain that

$$
\begin{aligned}
& \left(\left.\frac{1}{|Q|} \int_{Q}|| T_{b}\left(f_{1}, f_{2}\right)(z)\right|^{\delta}-|c|^{\delta} \mid d z\right)^{\frac{1}{\delta}} \\
& \quad \leq\left(\frac{1}{|Q|} \int_{Q}\left|T_{b}\left(f_{1}, f_{2}\right)(z)-c\right|^{\delta} d z\right)^{\frac{1}{\delta}} \\
& \quad \leq\left(\frac{1}{|Q|} \int_{Q}\left|(b(z)-\lambda) T\left(f_{1}, f_{2}\right)(z)\right|^{\delta} d z\right)^{\frac{1}{\delta}}+\left(\frac{1}{|Q|} \int_{Q}\left|T\left((b-\lambda) f_{1}, f_{2}\right)(z)-c\right|^{\delta} d z\right)^{\frac{1}{\delta}} \\
& \quad=:\left(P_{1}+P_{2}\right) .
\end{aligned}
$$


By Hölder's inequality we get

$$
\begin{aligned}
P_{1} & \leq C\|Q\|_{\dot{\lambda}_{\beta}}\left(\frac{1}{|Q|^{1-\frac{\delta \beta}{n}}} \int_{Q}\left|T\left(f_{1}, f_{2}\right)(z)\right|^{\delta} d z\right)^{\frac{1}{\delta}} \\
& \leq C\|b\|_{\dot{\lambda}_{\beta}} M_{\epsilon, \beta}\left(T\left(f_{1}, f_{2}\right)\right)(x) .
\end{aligned}
$$

We bound the second part as

$$
P_{2} \leq P_{21}+P_{22}+P_{23}+P_{24}
$$

where

$$
\begin{aligned}
& P_{21}=\left(\frac{1}{|Q|} \int_{Q}\left|T\left((b-\lambda) f_{1}^{0}, f_{2}^{0}\right)(z)\right|^{\delta} d x\right)^{\frac{1}{\delta}}, \\
& P_{22}=\left(\frac{1}{|Q|} \int_{Q}\left|T\left((b-\lambda) f_{1}^{0}, f_{2}^{\infty}\right)(z)\right|^{\delta} d z\right)^{\frac{1}{\delta}}, \\
& P_{23}=\left(\frac{1}{|Q|} \int_{Q}\left|T\left((b-\lambda) f_{1}^{\infty}, f_{2}^{0}\right)(z)\right|^{\delta} d z\right)^{\frac{1}{\delta}},
\end{aligned}
$$

and

$$
P_{24}=\left(\frac{1}{|Q|} \int_{Q}\left|T\left((b-\lambda) f_{1}^{\infty}, f_{2}^{\infty}\right)(z)-T\left((b-\lambda) f_{1}^{\infty}, f_{2}^{\infty}\right)(x)\right|^{\delta} d z\right)^{\frac{1}{\delta}}
$$

By Kolmogorov's inequality and Lemma 2.3 we get

$$
\begin{aligned}
P_{21} & \leq C\left\|T\left((b-\lambda) f_{1}^{0}, f_{2}^{0}\right)\right\|_{L^{1 / 2, \infty}\left(B, \frac{d x}{|Q|}\right)} \\
& \leq \frac{C}{|Q|} \int_{Q}\left|(b-\lambda) f_{1}^{0}(z)\right| d z \frac{1}{|Q|} \int_{Q}\left|f_{2}^{0}(z)\right| d z \\
& \leq C\|b\|_{\dot{\lambda}_{\beta}}\left|Q^{*}\right|^{\beta / n} \frac{1}{|Q|} \int_{Q}\left|f_{1}^{0}(z)\right| d z \frac{1}{|Q|} \int_{Q}\left|f_{2}^{0}(z)\right| d z \\
& \leq C\|b\|_{\dot{\lambda}_{\beta}} M_{1, \beta}\left(f_{1}\right)(x) M\left(f_{2}\right)(x) .
\end{aligned}
$$

By using the Minkowski inequality and (1.1) and (3.3) we obtain that

$$
\begin{aligned}
P_{22}= & \left(\frac{1}{|Q|} \int_{Q}\left|T\left((b-\lambda) f_{1}^{0}, f_{2}^{\infty}\right)(z)\right|^{\delta} d z\right)^{\frac{1}{\delta}} \\
\leq & C \frac{1}{|Q|} \int_{Q}\left|T\left((b-\lambda) f_{1}^{0}, f_{2}^{\infty}\right)(z)\right| d z \\
\leq & C \frac{1}{|Q|} \int_{Q} \int_{Q^{*}} \int_{\left(Q^{*}\right)^{c}}\left(\int_{0}^{\infty}\left|K_{t}\left(z, y_{1}, y_{2}\right)\right|^{2} \frac{d t}{t}\right)^{1 / 2} \\
& \times\left|\left(b\left(y_{1}\right)-\lambda\right) f_{1}\left(y_{1}\right)\right| f_{2}\left(y_{2}\right) \mid d y_{2} d y_{1} d z \\
\leq & C \frac{1}{|Q|} \int_{Q} \int_{Q^{*}} \int_{\left(Q^{*}\right)^{c}} \frac{1}{\left(\left|z-y_{1}\right|+\left|z-y_{2}\right|\right)^{2 n}}\left|\left(b\left(y_{1}\right)-\lambda\right) f_{1}\left(y_{1}\right)\right|\left|f_{2}\left(y_{2}\right)\right| d y_{2} d y_{1} d z
\end{aligned}
$$




$$
\begin{aligned}
& \leq C \int_{Q^{*}}\left|\left(b\left(y_{1}\right)-\lambda\right) f_{1}\left(y_{1}\right)\right| d y_{1} \int_{\mathbb{R}^{n} \backslash Q^{*}} \frac{\left|f_{2}\left(y_{2}\right)\right| d y_{2}}{\left|z-y_{2}\right|^{2 n}} \\
& \leq C\|b\|_{\dot{\lambda}_{\beta}} \int_{Q^{*}}\left|y_{1}-x_{Q}\right|^{\beta}\left|f_{1}\left(y_{1}\right)\right| d y_{1} \sum_{k=1}^{\infty} \int_{2^{k+1} Q^{*} \mid 2^{k} Q^{*}} \frac{\left|f_{2}\left(y_{2}\right)\right| d y_{2}}{\left|z-y_{2}\right|^{2 n}} \\
& \leq C\|b\|_{\dot{\lambda}_{\beta}} \sum_{k=1}^{\infty}\left|2^{k} Q^{*}\right|^{-2}\left|Q^{*}\right| \int_{2^{k+1} Q^{*} \mid 2^{k} Q^{*}}\left|f_{2}\left(y_{2}\right)\right| d y_{2} M_{1, \beta}\left(f_{1}\right)(x) \\
& \leq C\|b\|_{\dot{\lambda}_{\beta}} \sum_{k=1}^{\infty} 2^{-k} \frac{1}{\left|2^{k} Q^{*}\right|} \int_{2^{k+1} Q^{*}}\left|f_{2}\left(y_{2}\right)\right| d y_{2} M_{1, \beta}\left(f_{1}\right)(x) \\
& \leq C\|b\|_{\dot{\lambda}_{\beta}} M_{1, \beta}\left(f_{1}\right)(x) M\left(f_{2}\right)(x) .
\end{aligned}
$$

Similarly, we deduce that

$$
\begin{aligned}
& P_{23}=\left(\frac{1}{|Q|} \int_{Q}\left|T\left((b-\lambda) f_{1}^{\infty}, f_{2}^{0}\right)(z)\right|^{\delta} d z\right)^{\frac{1}{\delta}} \\
& \leq \frac{C}{|Q|} \int_{Q}\left|T\left((b-\lambda) f_{1}^{\infty}, f_{2}^{0}\right)(z)\right| d z \\
& \leq \frac{C}{|Q|} \int_{Q} \int_{Q^{*}} \int_{\left(Q^{*}\right)^{c}}\left(\int_{0}^{\infty}\left|K_{t}\left(z, y_{1}, y_{2}\right)\right|^{2} \frac{d t}{t}\right)^{1 / 2}\left|\left(b\left(y_{1}\right)-\lambda\right) f_{1}\left(y_{1}\right)\right|\left|f_{2}\left(y_{2}\right)\right| d y_{2} d y_{1} d z \\
& \leq \frac{C}{|Q|} \int_{Q} \int_{Q^{*}} \int_{\left(Q^{*}\right)^{c}} \frac{1}{\left(\left|z-y_{1}\right|+\left|z-y_{2}\right|\right)^{2 n}}\left|\left(b\left(y_{1}\right)-\lambda\right) f_{1}\left(y_{1}\right)\right|\left|f_{2}\left(y_{2}\right)\right| d y_{2} d y_{1} d z \\
& \leq C \int_{\left(Q^{*}\right)^{c}} \frac{\left|\left(b\left(y_{1}\right)-\lambda\right) f_{1}\left(y_{1}\right)\right| d y_{1}}{\left|y_{1}-x_{Q}\right|^{2 n}} \int_{Q^{*}}\left|f_{2}\left(y_{2}\right)\right| d y_{2} \\
& \leq C\left|Q^{*}\right| \sum_{k=1}^{\infty} \int_{2^{k+1} Q^{*} \mid 2^{k} Q^{*}} \frac{\left|\left(b\left(y_{1}\right)-\lambda\right) f_{1}\left(y_{1}\right)\right| d y_{1}}{\left|y_{1}-x_{Q}\right|^{2 n}} M\left(f_{2}\right)(x) \\
& \leq C\|b\|_{\dot{\lambda}_{\beta}}\left|Q^{*}\right| \sum_{k=1}^{\infty} \int_{2^{k+1} Q^{*} \mid 2^{k} Q^{*}}\left|y_{1}-x_{Q}\right|^{\beta-2 n}\left|f_{1}\left(y_{1}\right)\right| d y_{1} M\left(f_{2}\right)(x) \\
& \leq C\|b\|_{\lambda_{\beta}}\left|Q^{*}\right| \sum_{k=1}^{\infty}\left|2^{k} Q^{*}\right|^{\beta / n-2} \int_{2^{k+1} Q^{*} \backslash 2^{k} Q^{*}}\left|f_{1}\left(y_{1}\right)\right| d y_{1} M\left(f_{2}\right)(x) \\
& \leq C\|b\|_{\lambda_{\beta}} \sum_{k=1}^{\infty} 2^{-k} \frac{1}{\left|2^{k} Q^{*}\right|^{1-\beta / n}} \int_{2^{k+1} Q^{*}}\left|f_{1}\left(y_{1}\right)\right| d y_{1} M\left(f_{2}\right)(x) \\
& \leq C\|b\|_{\dot{\lambda}_{\beta}} M_{1, \beta}\left(f_{1}\right)(x) M\left(f_{2}\right)(x) \text {. }
\end{aligned}
$$

Since $\left(\mathbb{R}^{n} \backslash Q^{*}\right)^{2} \subseteq \mathbb{R}^{2 n} \backslash\left(Q^{*}\right)^{2} \subseteq \bigcup_{k=1}^{\infty}\left(2^{k+3} \sqrt{n} Q\right)^{2} \backslash\left(2^{k+2} \sqrt{n} Q\right)^{2}$, we can use Minkowski's inequality and (1.2) and (3.3) to get

$$
\begin{aligned}
P_{24} & \leq \frac{C}{|Q|} \int_{Q}\left|T\left((b-\lambda) f_{1}^{\infty}, f_{2}^{\infty}\right)(z)-T\left((b-\lambda) f_{1}^{\infty}, f_{2}^{\infty}\right)(x)\right| d z \\
& \leq \frac{C}{|Q|} \int_{Q} \int_{\left(\mathbb{R}^{n} \backslash Q^{*}\right)^{2}}\left(\int_{0}^{\infty}\left|K_{t}(z, \vec{y})-K_{t}(x, \vec{y})\right|^{2} \frac{d t}{t}\right)^{1 / 2}
\end{aligned}
$$




$$
\begin{aligned}
& \times\left|\left(b\left(y_{1}\right)-\lambda\right) \prod_{i=1}^{2} f_{i}^{\infty}\left(y_{i}\right)\right| d \vec{y} d z \\
\leq & \frac{C}{|Q|} \int_{Q} \int_{\left(\mathbb{R}^{n} \backslash Q^{*}\right)^{2}} \frac{1}{\left(\left|x-y_{1}\right|+\left|x-y_{2}\right|\right)^{2 n}} \omega\left(\frac{\left|z-x_{Q}\right|}{\left|x-y_{1}\right|+\left|x-y_{2}\right|}\right) \\
& \times\left|\left(b\left(y_{1}\right)-\lambda\right) \prod_{i=1}^{2} f_{i}^{\infty}\left(y_{i}\right)\right| d \vec{y} d z \\
\leq & \frac{C}{|Q|} \int_{Q} \sum_{k=1}^{\infty} \int_{\left(2^{k+3} \sqrt{n} Q \backslash 2^{k+2} \sqrt{n} Q\right)^{2}} \frac{1}{\left(\left|2^{k+3} \sqrt{n} Q\right|\right)^{2}} \omega\left(2^{-k}\right) \\
& \times\left|\left(b\left(y_{1}\right)-\lambda\right) \prod_{i=1}^{2} f_{i}^{\infty}\left(y_{i}\right)\right| d \vec{y} d z \\
\leq & C \frac{\|Q\| \dot{\lambda}_{\beta}}{|Q|} \int_{Q} \sum_{k=1}^{\infty} \frac{1}{\left(\left|2^{k+3} \sqrt{n} Q\right|\right)^{2}} \int_{\left(2^{k+3} \sqrt{n} Q \backslash 2^{k+2} \sqrt{n} Q\right)^{2}} \omega\left(2^{-k}\right)\left|y_{1}-x_{Q}\right|^{\beta} \\
& \times \prod_{i=1}^{2}\left|f_{i}^{\infty}\left(y_{i}\right)\right| d \vec{y} d z \\
\leq & C\|b\|_{\dot{\lambda}_{\beta}} \sum_{k=1}^{\infty} \frac{\omega\left(2^{-k}\right)}{\left(\left|2^{k+3} \sqrt{n} Q\right|\right)^{1-\beta / n}} \int_{2^{k+3} \sqrt{n} Q}\left|f_{1}^{\infty}\left(y_{1}\right)\right| d y_{1} \frac{1}{\left|2^{k} Q^{*}\right|} \int_{2^{k+3} \sqrt{n} Q}\left|f_{2}^{\infty}\left(y_{2}\right)\right| d y_{2} \\
\leq & C\|b\|_{\dot{\beta}_{\beta}} M_{1, \beta_{1}}\left(f_{1}\right)(x) M\left(f_{2}\right)(x) .
\end{aligned}
$$

Thus we finish the proof of (3.2). Then Lemma 3.1 is proved.

Proofs of Theorem 1.1 By using Lemma 3.1 and modifying the proof of Theorem 1.1 in [8] we can finish the proof of Theorem 1.1. We omit the proof.

\section{Proof of Theorems $\mathbf{1 . 2}$ and 1.3}

For simplicity, we just consider the case $m=2$; our method still holds for general $m$ with little modifications.

Proof of Theorem 1.2 The theorem will be proved if we show that

$$
\sup _{Q} \frac{1}{|Q|^{1+\beta / n-1 / p}} \int_{Q}\left|T_{\Pi \vec{b}}(\vec{f})(z)-\left(T_{\Pi \vec{b}}(\vec{f})\right)_{Q}\right| d z \leq C\left\|b_{1}\right\|_{{\dot{\beta_{1}}}_{1}}\left\|b_{2}\right\|_{{\dot{\beta_{2}}}_{2}}\left\|f_{1}\right\|_{L^{p_{1}}}\left\|f_{2}\right\|_{L^{p_{2}}}
$$

Let $c=c_{1}+c_{2}+c_{3}$, which will be determined later. Then we have

$$
\begin{aligned}
\frac{1}{|Q|^{1+\beta / n-1 / p}} \int_{Q}\left|T_{\Pi \vec{b}}(\vec{f})(z)-\left(T_{\Pi \vec{b}}(\vec{f})\right)_{Q}\right| d z \\
\leq \frac{1}{|Q|^{1+\beta / n-1 / p}} \int_{Q}\left|T_{\Pi \vec{b}}\left(f_{1}, f_{2}\right)(z)-c\right| d z \\
\leq \frac{C}{|Q|^{1+\beta / n-1 / p}} \int_{Q}\left|T_{\Pi \vec{b}}\left(f_{1}^{0}, f_{2}^{0}\right)(z)\right| d z \\
\quad+\frac{C}{|Q|^{1+\beta / n-1 / p}} \int_{Q}\left|T_{\Pi \vec{b}}\left(f_{1}^{0}, f_{2}^{\infty}\right)(z)-c_{1}\right| d z
\end{aligned}
$$




$$
\begin{aligned}
& +\frac{C}{|Q|^{1+\beta / n-1 / p}} \int_{Q}\left|T_{\Pi \vec{b}}\left(f_{1}^{\infty}, f_{2}^{0}\right)(z)-c_{2}\right| d z \\
& +\frac{C}{|Q|^{1+\beta / n-1 / p}} \int_{Q}\left|T_{\Pi \vec{b}}\left(f_{1}^{\infty}, f_{2}^{\infty}\right)(z)-c_{3}\right| d z \\
\doteq & M_{1}+M_{2}+M_{3}+M_{4} .
\end{aligned}
$$

We can choose $1<q, q_{j}<\infty, q_{j}<n / \beta_{j}<p_{j}, j=1,2$, with $1 / q=1 / q_{1}+1 / q_{2}-\left(\beta_{1}+\beta_{2}\right) / n$. By Hölder's inequality and Theorem 1.1 we obtain

$$
\begin{aligned}
M_{1} & \leq \frac{C}{|Q|^{1+\beta / n-1 / p}}\left(\int_{Q}\left|T_{\Pi \vec{b}}\left(f_{1}^{0}, f_{2}^{0}\right)(z)\right|^{q} d z\right)^{1 / q}|Q|^{1-1 / q} \\
& \leq \frac{C}{|Q|^{1+\beta / n-1 / p}}|Q|^{1-1 / q}\left\|f_{1}^{0}\right\|_{L^{q_{1}}}\left\|f_{2}^{0}\right\|_{L^{q_{2}}} \\
& \leq C\left\|f_{1}\right\|_{L^{p_{1}}}\left\|f_{2}\right\|_{L^{p_{2}}} .
\end{aligned}
$$

For the second term, we take $c_{1}=T\left(\left(b_{1}-\lambda_{1}\right) f_{1}^{0}, f_{2}^{\infty}\right)\left(x_{Q}\right)$. Then

$$
\begin{aligned}
M_{2} \leq & \frac{C}{|Q|^{1+\beta / n-1 / p}} \int_{Q}\left(\int_{0}^{\infty} \mid \int_{Q^{*}} \int_{\mathbb{R}^{n} \backslash Q^{*}}\left(b_{1}(z)-\lambda_{1}\right)\left(b_{2}(z)-\lambda_{2}\right)\right. \\
& \left.\times\left. K_{t}\left(z, y_{1}, y_{2}\right) f_{1}\left(y_{1}\right) f_{2}\left(y_{2}\right) d y_{1} d y_{2}\right|^{2} \frac{d t}{t}\right)^{1 / 2} d z \\
& +\frac{C}{|Q|^{1+\beta / n-1 / p}} \int_{Q}\left(\int_{0}^{\infty} \mid \int_{Q^{*}} \int_{\mathbb{R}^{n} \backslash Q^{*}}\left(b_{1}(z)-\lambda_{1}\right)\left(b_{2}\left(y_{2}\right)-\lambda_{2}\right)\right. \\
& \left.\times\left. K_{t}\left(z, y_{1}, y_{2}\right) f_{1}\left(y_{1}\right) f_{2}\left(y_{2}\right) d y_{1} d y_{2}\right|^{2} \frac{d t}{t}\right)^{1 / 2} d z \\
& +\frac{C}{|Q|^{1+\beta / n-1 / p}} \int_{Q}\left(\int_{0}^{\infty} \mid \int_{Q^{*}} \int_{\mathbb{R}^{n} \backslash Q^{*}}\left(b_{1}\left(y_{1}\right)-\lambda_{1}\right)\left(b_{2}(z)-\lambda_{2}\right)\right. \\
& \left.\times\left. K_{t}\left(z, y_{1}, y_{2}\right) f_{1}\left(y_{1}\right) f_{2}\left(y_{2}\right) d y_{1} d y_{2}\right|^{2} \frac{d t}{t}\right)^{1 / 2} d z \\
& +\frac{C}{|Q|^{1+\beta / n-1 / p}} \int_{Q}\left(\int_{0}^{\infty} \mid \int_{Q^{*}} \int_{\mathbb{R}^{n} \backslash Q^{*}}\left(b_{1}\left(y_{1}\right)-\lambda_{1}\right)\left(b_{2}\left(y_{2}\right)-\lambda_{2}\right)\right. \\
& \left.\times\left.\left[K_{t}\left(z, y_{1}, y_{2}\right)-K_{t}\left(x_{Q}, y_{1}, y_{2}\right)\right] f_{1}\left(y_{1}\right) f_{2}\left(y_{2}\right) d y_{1} d y_{2}\right|^{2} \frac{d t}{t}\right)^{1 / 2} d z \\
\doteq & M_{21}+M_{22}+M_{23}+M_{24} .
\end{aligned}
$$

By Minkowski's inequality and the size condition (1.1) we have

$$
\begin{aligned}
M_{21} \leq & \frac{C}{|Q|^{1+\beta / n-1 / p}} \int_{Q} \int_{Q^{*}} \int_{\mathbb{R}^{n} \backslash Q^{*}}\left|\left(b_{1}(z)-\lambda_{1}\right)\left(b_{2}(z)-\lambda_{2}\right)\right| \\
& \times\left(\int_{0}^{\infty}\left|K_{t}\left(z, y_{1}, y_{2}\right)\right|^{2} \frac{d t}{t}\right)^{1 / 2}\left|f_{1}\left(y_{1}\right) f_{2}\left(y_{2}\right)\right| d y_{1} d y_{2} d z \\
\leq & \frac{C}{|Q|^{1+\beta / n-1 / p}} \int_{Q} \int_{Q^{*}} \int_{\mathbb{R}^{n} \backslash Q^{*}}\left|\left(b_{1}(z)-\lambda_{1}\right)\left(b_{2}(z)-\lambda_{2}\right)\right| \\
& \times \frac{1}{\left(\left|z-y_{1}\right|+\left|z-y_{2}\right|\right)^{2 n}}\left|f_{1}\left(y_{1}\right) f_{2}\left(y_{2}\right)\right| d y_{1} d y_{2} d z
\end{aligned}
$$




$$
\begin{aligned}
& \leq \frac{C}{|Q|^{1+\beta / n-1 / p}} \int_{Q}\left|\left(b_{1}(z)-\lambda_{1}\right)\left(b_{2}(z)-\lambda_{2}\right)\right| \\
& \times \int_{Q^{*}} \int_{\mathbb{R}^{n} \backslash Q^{*}} \frac{1}{\left(\left|z-y_{1}\right|+\left|z-y_{2}\right|\right)^{2 n}}\left|f_{1}\left(y_{1}\right) f_{2}\left(y_{2}\right)\right| d y_{1} d y_{2} d z \\
& \leq\left\|b_{1}\right\|_{{\dot{\beta_{1}}}_{1}}\left\|b_{2}\right\|_{{\dot{\beta_{2}}}_{2}}|Q|^{1 / p} \int_{Q^{*}}\left|f_{1}\left(y_{1}\right)\right| d y_{1} \\
& \times \sum_{k=1}^{\infty} \int_{2^{k+3} \sqrt{n} Q \backslash 2^{k+2} \sqrt{n} Q} \frac{\left|f_{2}\left(y_{2}\right)\right|}{\left|y_{2}-x_{Q}\right|^{2 n}} d y_{2}
\end{aligned}
$$

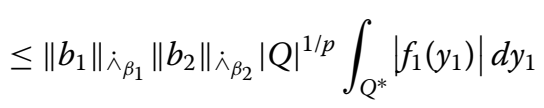

$$
\begin{aligned}
& \times \sum_{k=1}^{\infty} \frac{1}{\left|2^{k+3} \sqrt{n} Q\right|^{2}} \int_{2^{k+3} \sqrt{n} Q \backslash 2^{k+2} \sqrt{n} Q}\left|f_{2}\left(y_{2}\right)\right| d y_{2} \\
& \leq\left\|b_{1}\right\|{\lambda_{\beta_{1}}}\left\|b_{2}\right\|_{\lambda_{\beta_{2}}}\left\|f_{1}\right\|_{L^{p_{1}}}\left\|f_{2}\right\|_{L^{p_{2}}} \sum_{k=1}^{\infty} 2^{k n\left(-1-1 / p_{2}\right)} \\
& \leq\left\|b_{1}\right\|_{{\dot{\beta_{1}}}_{1}}\left\|b_{2}\right\|_{{\dot{\beta_{2}}}_{\beta_{2}}}\left\|f_{1}\right\|_{L^{p_{1}}}\left\|f_{2}\right\|_{L^{p_{2}}}
\end{aligned}
$$

We now proceed as in the estimate of $M_{21}$ :

$$
\begin{aligned}
& M_{22} \leq \frac{C}{|Q|^{1+\beta / n-1 / p}} \int_{Q} \int_{Q^{*}} \int_{\mathbb{R}^{n} \backslash Q^{*}}\left|\left(b_{1}(z)-\lambda_{1}\right)\left(b_{2}\left(y_{2}\right)-\lambda_{2}\right)\right| \\
& \times\left(\int_{0}^{\infty}\left|K_{t}\left(z, y_{1}, y_{2}\right)\right|^{2} \frac{d t}{t}\right)^{1 / 2}\left|f_{1}\left(y_{1}\right) f_{2}\left(y_{2}\right)\right| d y_{1} d y_{2} d z \\
& \leq \frac{C}{|Q|^{1+\beta / n-1 / p}} \int_{Q} \int_{Q^{*}} \int_{\mathbb{R}^{n} \backslash Q^{*}}\left|\left(b_{1}(z)-\lambda_{1}\right)\left(b_{2}\left(y_{2}\right)-\lambda_{2}\right)\right| \\
& \times \frac{1}{\left(\left|z-y_{1}\right|+\left|z-y_{2}\right|\right)^{2 n}}\left|f_{1}\left(y_{1}\right) f_{2}\left(y_{2}\right)\right| d y_{1} d y_{2} d z \\
& \leq \frac{C}{|Q|^{1+\beta / n-1 / p}} \int_{Q}\left|\left(b_{1}(z)-\lambda_{1}\right)\right| \\
& \times \int_{Q^{*}} \int_{\mathbb{R}^{n} \backslash Q^{*}} \frac{\left|\left(b_{2}(z)-\lambda_{2}\right)\right|}{\left(\left|z-y_{1}\right|+\left|z-y_{2}\right|\right)^{2 n}}\left|f_{1}\left(y_{1}\right) f_{2}\left(y_{2}\right)\right| d y_{1} d y_{2} d z \\
& \leq \frac{\left\|b_{1}\right\|{\dot{\lambda_{1}}}_{\beta_{1}}\left\|b_{2}\right\|_{\dot{\beta}_{\beta_{2}}}}{|Q|^{\beta_{2} / n-1 / p}} \int_{Q^{*}}\left|f_{1}\left(y_{1}\right)\right| d y_{1} \sum_{k=1}^{\infty} \int_{2^{k+3} \sqrt{n} Q \backslash 2^{k+2} \sqrt{n} Q} \frac{\left|f_{2}\left(y_{2}\right)\right|}{\left|y_{2}-x_{Q}\right|^{2 n-\beta_{2}}} d y_{2} \\
& \leq\left\|b_{1}\right\|{{\dot{\beta_{1}}}_{\beta_{1}}}\left\|b_{2}\right\|_{{\dot{\beta_{2}}}_{2}}\left\|f_{1}\right\|_{L^{p_{1}}}\left\|f_{2}\right\|_{L^{p_{2}}} \sum_{k=1}^{\infty} 2^{k n\left(-1-1 / p_{2}+\beta_{2} / n\right)} \\
& \leq\left\|b_{1}\right\|{\dot{\lambda_{\beta_{1}}}}_{\| b_{2}}\left\|{{\dot{\lambda_{\beta}}}_{\beta_{2}}}\right\| f_{1}\left\|_{L^{p_{1}}}\right\| f_{2} \|_{L^{p_{2}}}
\end{aligned}
$$

because of $-1-1 / p_{2}+\beta_{2} / n<0$.

Similarly,

$M_{23} \leq C\left\|b_{1}\right\|_{\dot{\lambda}_{\beta_{1}}}\left\|b_{2}\right\|_{{\dot{\lambda_{\beta}}}_{\beta_{2}}}\left\|f_{1}\right\|_{L^{p_{1}}}\left\|f_{2}\right\|_{L^{p_{2}}}$. 
By Minkowski’s inequality and (1.2) we have

$$
\begin{aligned}
& M_{24} \leq \frac{C}{|Q|^{1+\beta / n-1 / p}} \int_{Q} \int_{Q^{*}} \int_{\mathbb{R}^{n} \backslash Q^{*}}\left|\left(b_{1}(z)-\lambda_{1}\right)\left(b_{2}\left(y_{2}\right)-\lambda_{2}\right)\right| \\
& \times\left(\int_{0}^{\infty}\left|K_{t}\left(z, y_{1}, y_{2}\right)-K_{t}\left(x_{Q}, y_{1}, y_{2}\right)\right|^{2} \frac{d t}{t}\right)^{1 / 2}\left|f_{1}\left(y_{1}\right) f_{2}\left(y_{2}\right)\right| d y_{1} d y_{2} d z \\
& \leq C\left\|b_{1}\right\|{\dot{\dot{\lambda}_{\beta}}}_{\beta_{1}}\left\|b_{2}\right\|_{\dot{\wedge}_{\beta_{2}}} \frac{1}{|Q|^{1+\beta / n-1 / p}} \int_{Q} \int_{Q^{*}} \int_{\mathbb{R}^{n} \backslash Q^{*}}\left|\left(b_{1}(z)-\lambda_{1}\right)\left(b_{2}\left(y_{2}\right)-\lambda_{2}\right)\right| \\
& \times \frac{\omega\left(\frac{\left|z-x_{Q}\right|}{\left|z-y_{1}\right|+\left|z-y_{2}\right|}\right)}{\left(\left|z-y_{1}\right|+\left|z-y_{2}\right|\right)^{2 n}}\left|f_{1}\left(y_{1}\right) f_{2}\left(y_{2}\right)\right| d y_{1} d y_{2} d z \\
& \leq C\left\|b_{1}\right\|_{\dot{\lambda}_{\beta_{1}}}\left\|b_{2}\right\|_{\dot{\lambda}_{\beta_{2}}} \frac{1}{|Q|^{1+\beta_{2} / n-1 / p}} \int_{Q} \int_{Q^{*}} \int_{\mathbb{R}^{n} \backslash Q^{*}}\left|\left(b_{1}(z)-\lambda_{1}\right)\left(b_{2}\left(y_{2}\right)-\lambda_{2}\right)\right| \\
& \times \frac{\omega\left(\frac{\left|z-x_{Q}\right|}{\left|x_{Q}-y_{2}\right|}\right)}{\left(\left|z-y_{1}\right|+\left|z-y_{2}\right|\right)^{2 n-\beta_{2}}}\left|f_{1}\left(y_{1}\right) f_{2}\left(y_{2}\right)\right| d y_{1} d y_{2} d z \\
& \leq C\left\|b_{1}\right\|_{\dot{\lambda}_{\beta_{1}}}\left\|b_{2}\right\|_{\dot{\lambda}_{\beta_{2}}} \frac{C}{|Q|^{\beta_{2} / n-1 / p}} \int_{Q}\left|\left(b_{1}(z)-\lambda_{1}\right)\right| \\
& \times \int_{Q^{*}} \int_{\mathbb{R}^{n} \backslash Q^{*}} \frac{\left|\left(b_{2}(z)-\lambda_{2}\right)\right|}{\left(\left|z-y_{1}\right|+\left|z-y_{2}\right|\right)^{2 n}}\left|f_{1}\left(y_{1}\right) f_{2}\left(y_{2}\right)\right| d y_{1} d y_{2} d z \\
& \leq C \frac{\left\|b_{1}\right\|{\dot{\lambda_{\beta_{1}}}}\left\|b_{2}\right\|{\dot{\lambda_{\beta_{2}}}}_{\mathrm{Q}}}{|Q|^{\beta_{2} / n-1 / p}} \int_{Q^{*}}\left|f_{1}\left(y_{1}\right)\right| d y_{1} \\
& \times \sum_{k=1}^{\infty} \frac{\omega\left(2^{-k}\right)}{\left|2^{k+3} \sqrt{n} Q\right|^{2-\beta_{2} / n}} \int_{2^{k+3} \sqrt{n} Q \backslash 2^{k+2} \sqrt{n} Q}\left|f_{2}\left(y_{2}\right)\right| d y_{2} \\
& \leq C\left\|b_{1}\right\|_{\dot{\lambda}_{\beta_{1}}}\left\|b_{2}\right\|_{{\dot{\beta_{\beta}}}_{\beta_{2}}}\left\|f_{1}\right\|_{L^{p_{1}}}\left\|f_{2}\right\|_{L^{p_{2}}} \sum_{k=1}^{\infty} \omega\left(2^{-k}\right) 2^{k n\left(1-\beta_{2} / n+1 / p_{2}\right)} \\
& \leq C\left\|b_{1}\right\|_{\dot{\lambda}_{\beta_{1}}}\left\|b_{2}\right\|_{\dot{\lambda}_{\beta_{2}}}\left\|f_{1}\right\|_{L^{p_{1}}}\left\|f_{2}\right\|_{L^{p_{2}}},
\end{aligned}
$$

where we have used the fact $1-\beta_{2} / n+1 / p_{2}>0$.

Thus

$$
M_{2} \leq C\left\|b_{1}\right\|_{\dot{\lambda}_{\beta_{1}}}\left\|b_{2}\right\|_{\dot{\lambda}_{\beta_{2}}}\left\|f_{1}\right\|_{L^{p_{1}}}\left\|f_{2}\right\|_{L^{p_{2}}}
$$

Similarly,

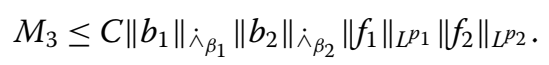

We deal with $M_{4}$ as follows:

$$
\begin{aligned}
M_{4} \leq & \frac{C}{|Q|^{1+\beta / n-1 / p}} \int_{Q}\left(\int_{0}^{\infty} \mid \int_{\left(\mathbb{R}^{n} \backslash Q^{*}\right)^{2}}\left(b_{1}(z)-\lambda_{1}\right)\left(b_{2}(z)-\lambda_{2}\right)\right. \\
& \left.\times\left. K_{t}\left(z, y_{1}, y_{2}\right) f_{1}\left(y_{1}\right) f_{2}\left(y_{2}\right) d y_{1} d y_{2}\right|^{2} \frac{d t}{t}\right)^{1 / 2} d z \\
& +\frac{C}{|Q|^{1+\beta / n-1 / p}} \int_{Q}\left(\int_{0}^{\infty} \mid \int_{\left(\mathbb{R}^{n} \backslash Q^{*}\right)^{2}}\left(b_{1}(z)-\lambda_{1}\right)\left(b_{2}\left(y_{2}\right)-\lambda_{2}\right)\right.
\end{aligned}
$$




$$
\begin{aligned}
& \left.\times\left.\left[K_{t}\left(z, y_{1}, y_{2}\right)-K_{t}\left(x_{Q}, y_{1}, y_{2}\right)\right] f_{1}\left(y_{1}\right) f_{2}\left(y_{2}\right) d y_{1} d y_{2}\right|^{2} \frac{d t}{t}\right)^{1 / 2} d z \\
& +\frac{C}{|Q|^{1+\beta / n-1 / p}} \int_{Q}\left(\int_{0}^{\infty} \mid \int_{\left(\mathbb{R}^{n} \backslash Q^{*}\right)^{2}}\left(b_{1}\left(y_{1}\right)-\lambda_{1}\right)\left(b_{2}(z)-\lambda_{2}\right)\right. \\
& \left.\times\left.\left[K_{t}\left(z, y_{1}, y_{2}\right)-K_{t}\left(x_{Q}, y_{1}, y_{2}\right)\right] f_{1}\left(y_{1}\right) f_{2}\left(y_{2}\right) d y_{1} d y_{2}\right|^{2} \frac{d t}{t}\right)^{1 / 2} d z \\
& +\frac{C}{|Q|^{1+\beta / n-1 / p}} \int_{Q}\left(\int_{0}^{\infty} \mid \int_{\left(\mathbb{R}^{n} \backslash Q^{*}\right)^{2}}\left(b_{1}\left(y_{1}\right)-\lambda_{1}\right)\left(b_{2}\left(y_{2}\right)-\lambda_{2}\right)\right. \\
& \left.\times\left.\left[K_{t}\left(z, y_{1}, y_{2}\right)-K_{t}\left(x_{Q}, y_{1}, y_{2}\right)\right] f_{1}\left(y_{1}\right) f_{2}\left(y_{2}\right) d y_{1} d y_{2}\right|^{2} \frac{d t}{t}\right)^{1 / 2} d z \\
\doteq & M_{41}+M_{42}+M_{43}+M_{44} .
\end{aligned}
$$

By Minkowski's inequality and the size condition (1.1) we have

$$
\begin{aligned}
M_{41} \leq & \frac{C}{|Q|^{1+\beta / n-1 / p}} \int_{Q} \int_{\left(\mathbb{R}^{n} \backslash Q^{*}\right)^{2}}\left|\left(b_{1}(z)-\lambda_{1}\right)\left(b_{2}(z)-\lambda_{2}\right)\right| \\
& \times\left(\int_{0}^{\infty}\left|K_{t}\left(z, y_{1}, y_{2}\right)\right|^{2} \frac{d t}{t}\right)^{1 / 2}\left|f_{1}\left(y_{1}\right) f_{2}\left(y_{2}\right)\right| d y_{1} d y_{2} d z \\
\leq & \frac{C}{|Q|^{1+\beta / n-1 / p}} \int_{Q} \int_{\left(\mathbb{R}^{n} \backslash Q^{*}\right)^{2}}\left|\left(b_{1}(z)-\lambda_{1}\right)\left(b_{2}(z)-\lambda_{2}\right)\right| \\
& \times \frac{1}{\left(\left|z-y_{1}\right|+\left|z-y_{2}\right|\right)^{2 n}}\left|f_{1}\left(y_{1}\right) f_{2}\left(y_{2}\right)\right| d y_{1} d y_{2} d z \\
\leq & \left\|b_{1}\right\|_{\dot{\lambda}_{\beta_{1}}}\left\|b_{2}\right\|_{\dot{\lambda}_{\beta_{2}}}|Q|^{1 / p} \sum_{k=1}^{\infty} \int_{2^{k+3} \sqrt{n} Q \backslash 2^{k+2} \sqrt{n} Q} \frac{\left|f_{1}\left(y_{1}\right)\right|}{\left|y_{1}-x_{Q}\right|^{n}} d y_{1} \\
& \times \sum_{k=1}^{\infty} \int_{2^{k+3} \sqrt{n} Q 2^{k+2} \sqrt{n} Q} \frac{\left|f_{2}\left(y_{2}\right)\right|}{\left|y_{2}-x_{Q}\right|^{n}} d y_{2} \\
\leq & \left\|b_{1}\right\|_{\dot{\beta}_{\beta_{1}}}\left\|b_{2}\right\|_{\dot{\lambda}_{\beta_{2}}}\left\|f_{1}\right\|_{L^{p_{1}}}\left\|f_{2}\right\|_{L^{p_{2}}|Q|^{1 / p}} \sum_{k=1}^{\infty}\left|2^{k+3} \sqrt{n} Q\right|^{-1 / p_{1}} \sum_{k=1}^{\infty}\left|2^{k+3} \sqrt{n} Q\right|^{-1 / p_{2}} \\
\leq & \left\|b_{1}\right\|_{\dot{\lambda}_{1}}\left\|b_{2}\right\|_{\dot{\lambda}_{\beta_{2}}}\left\|f_{1}\right\|_{L^{p_{1}}}\left\|f_{2}\right\|_{L^{p_{2}} .} .
\end{aligned}
$$

By Minkowski's inequality and the smooth condition (1.2) we have

$$
\begin{aligned}
M_{42} \leq & \frac{C}{|Q|^{1+\beta / n-1 / p}} \int_{Q} \int_{\left(\mathbb{R}^{n} \backslash Q^{*}\right)^{2}}\left|\left(b_{1}(z)-\lambda_{1}\right)\left(b_{2}\left(y_{2}\right)-\lambda_{2}\right)\right| \\
& \times\left(\int_{0}^{\infty}\left|K_{t}\left(z, y_{1}, y_{2}\right)-K_{t}\left(x_{Q}, y_{1}, y_{2}\right)\right|^{2} \frac{d t}{t}\right)^{1 / 2}\left|f_{1}\left(y_{1}\right) f_{2}\left(y_{2}\right)\right| d y_{1} d y_{2} d z \\
\leq & \frac{\left\|b_{1}\right\|{\dot{\lambda_{1}}}_{\beta_{1}}\left\|b_{2}\right\|_{\dot{\lambda}_{\beta_{2}}}}{|Q|^{\beta_{2} / n-1 / p}} \sum_{k=1}^{\infty} \int_{2^{k+3} \sqrt{n} Q \backslash 2^{k+2} \sqrt{n} Q} \frac{\left|f_{1}\left(y_{1}\right)\right|}{\left|y_{1}-x_{Q}\right|^{n}} d y_{1} \\
& \times \sum_{i=1}^{\infty} \int_{2^{i+3} \sqrt{n} Q \backslash 2^{i+2} \sqrt{n} Q} \frac{\left|f_{2}\left(y_{2}\right)\right| \omega\left(2^{-i}\right)}{\left|y_{2}-x_{Q}\right|^{n-\beta_{2}}} d y_{2} \\
\leq & \left\|b_{1}\right\|_{\dot{\beta}_{\beta_{1}}}\left\|b_{2}\right\|_{\dot{\lambda}_{\beta_{2}}}\left\|f_{1}\right\|_{L^{p_{1}}}\left\|f_{2}\right\|_{L^{p_{2}}} \sum_{k=1}^{\infty}\left|2^{k+3} \sqrt{n} Q\right|^{-1 / p_{1}}|Q|^{1 / p}
\end{aligned}
$$




$$
\begin{aligned}
& \times \sum_{i=1}^{\infty} \omega\left(2^{-i}\right)\left|2^{i+3} \sqrt{n} Q\right|^{\beta_{2} / n-1 / p_{2}}|Q|^{-\beta_{2} / n+1 / p_{2}} \\
\leq & \left\|b_{1}\right\|{\dot{\lambda_{\beta_{1}}}}_{\| b_{2}}\left\|_{{\dot{\beta_{2}}}_{2}}\right\| f_{1}\left\|_{L^{p_{1}}}\right\| f_{2} \|_{L^{p_{2}}} \sum_{i=1}^{\infty} \omega\left(2^{-i}\right) 2^{i n(\beta / n-1 / p)} \\
\leq & \left\|b_{1}\right\|_{{\dot{\beta_{1}}}_{1}}\left\|b_{2}\right\|_{{\dot{\beta_{2}}}_{2}}\left\|f_{1}\right\|_{L^{p_{1}}}\left\|f_{2}\right\|_{L^{p_{2}}} .
\end{aligned}
$$

Similarly,

$$
M_{43} \leq C\left\|b_{1}\right\|_{{\dot{\beta_{1}}}_{1}}\left\|b_{2}\right\|_{{\dot{\lambda_{2}}}_{2}}\left\|f_{1}\right\|_{L^{p_{1}}}\left\|f_{2}\right\|_{L^{p_{2}}}
$$

Now we estimate $M_{44}$ :

$$
\begin{aligned}
M_{44} \leq & \frac{C}{|Q|^{1+\beta / n-1 / p}} \int_{Q} \int_{\left(\mathbb{R}^{n} \backslash Q^{*}\right)^{2}}\left|\left(b_{1}\left(y_{1}\right)-\lambda_{1}\right)\left(b_{2}\left(y_{2}\right)-\lambda_{2}\right)\right| \\
& \times\left(\int_{0}^{\infty}\left|K_{t}\left(z, y_{1}, y_{2}\right)-K_{t}\left(x_{Q}, y_{1}, y_{2}\right)\right|^{2} \frac{d t}{t}\right)^{1 / 2}\left|f_{1}\left(y_{1}\right) f_{2}\left(y_{2}\right)\right| d y_{1} d y_{2} d z \\
\leq & \frac{\left\|b_{1}\right\|_{\dot{\beta}_{1}}\left\|b_{2}\right\|{\dot{\lambda_{\beta_{2}}}}_{\mid}}{|Q|^{\beta / n-1 / p}} \sum_{k=1}^{\infty} \int_{\left(2^{k+3} \sqrt{n} Q\right)^{2} \backslash\left(2^{k+2} \sqrt{n} Q\right)^{2}} \frac{\left|f_{1}\left(y_{1}\right)\right|}{\left|y_{2}-x_{Q}\right|^{2 n-\beta_{1}-\beta_{2}}} \\
& \times \omega\left(\frac{\left|z-x_{Q}\right|}{\left|y_{2}-x_{Q}\right|}\right) d y_{1} d y_{2} \\
\leq & \left\|b_{1}\right\|_{\dot{\lambda}_{\beta_{1}}}\left\|b_{2}\right\|_{{\dot{\beta_{2}}}_{2}}\left\|f_{1}\right\|_{L^{p_{1}}}\left\|f_{2}\right\|_{L^{p_{2}}} \sum_{k=1}^{\infty} \omega\left(2^{-k}\right) 2^{k n(\beta / n-1 / p)} \\
\leq & \left\|b_{1}\right\|_{\dot{\lambda}_{\beta_{1}}}\left\|b_{2}\right\|_{{\dot{\beta_{2}}}_{2}}\left\|f_{1}\right\|_{L^{p_{1}}}\left\|f_{2}\right\|_{L^{p_{2}}} .
\end{aligned}
$$

Combing the estimates for $M_{1}, M_{2}, M_{3}, M_{4}$, we get (4.1). Thus the proof of Theorem 1.2 is completed.

Proof of Theorem 1.3 Let $c=c_{1}+c_{2}+c_{3}$, which will be determined later. Then we have

$$
\begin{aligned}
\left.\frac{1}{|Q|^{1+\beta / n}} \int_{Q} \mid T_{\Pi \vec{b}} \vec{f}\right)(z)-\left(T_{\Pi \vec{b}}(\vec{f})\right)_{Q} \mid d z \\
\leq \frac{1}{|Q|^{1+\beta / n}} \int_{Q}\left|T_{\Pi \vec{b}}\left(f_{1}, f_{2}\right)(z)-c\right| d z \\
\leq \frac{C}{|Q|^{1+\beta / n}} \int_{Q}\left|\left(b_{1}(z)-\lambda_{1}\right)\left(b_{2}(z)-\lambda_{2}\right) T\left(f_{1}, f_{2}\right)(z)\right| d z \\
\quad+\frac{C}{|Q|^{1+\beta / n}} \int_{Q}\left|\left(b_{2}(z)-\lambda_{2}\right) T_{\vec{b}}^{1}\left(f_{1}, f_{2}\right)(z)-c_{1}\right| d z \\
\quad+\frac{C}{|Q|^{1+\beta / n}} \int_{Q}\left|\left(b_{1}(z)-\lambda_{1}\right) T_{\vec{b}}^{2}\left(f_{1}, f_{2}\right)(z)-c_{2}\right| d z \\
\quad+\frac{C}{|Q|^{1+\beta / n}} \int_{Q}\left|T\left(\left(b_{1}-\lambda_{1}\right) f_{1},\left(b_{2}-\lambda_{2}\right) f_{2}\right)(z)-c_{3}\right| d z \\
\doteq N_{1}+N_{2}+N_{3}+N_{4} .
\end{aligned}
$$


In what follows, we estimate each term separately. For $1<r<p$, by the Hölder inequality we have

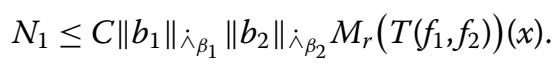

Observe that

$$
\begin{aligned}
& {\left[b_{1}, T\right]\left(f_{1}, f_{2}\right)(z)} \\
& <\left|\left(b_{1}(z)-\lambda_{1}\right)\right| T\left(f_{1}, f_{2}\right)(z)+T\left(f_{1}^{0}, f_{2}^{0}\right)(z) \\
& \quad+\left(\int_{0}^{\infty}\left|\int_{\left(\mathbb{R}^{n)^{m}}\right.}\left(b_{1}\left(y_{1}\right)-\lambda_{1}\right) K_{t}\left(x, y_{1}, y_{2}\right) f_{1}^{\infty}\left(y_{1}\right) f_{2}^{0}\left(y_{2}\right) d y_{1} d y_{2}\right|^{2} \frac{d t}{t}\right)^{\frac{1}{2}} \\
& \quad+\left(\int_{0}^{\infty}\left|\int_{\left(\mathbb{R}^{n)^{m}}\right.}\left(b_{1}\left(y_{1}\right)-\lambda_{1}\right) K_{t}\left(x, y_{1}, y_{2}\right) f_{1}^{0}\left(y_{1}\right) f_{2}^{\infty}\left(y_{2}\right) d y_{1} d y_{2}\right|^{2} \frac{d t}{t}\right)^{\frac{1}{2}} \\
& \quad+\left(\int_{0}^{\infty}\left|\int_{\left(\mathbb{R}^{n)^{m}}\right.}\left(b_{1}\left(y_{1}\right)-\lambda_{1}\right) K_{t}\left(x, y_{1}, y_{2}\right) f_{1}^{\infty}\left(y_{1}\right) f_{2}^{\infty}\left(y_{2}\right) d y_{1} d y_{2}\right|^{2} \frac{d t}{t}\right)^{\frac{1}{2}} .
\end{aligned}
$$

Let

$$
\begin{aligned}
c_{1}^{\prime}= & \left\|b_{2}\right\|_{\dot{\lambda}_{\beta_{2}}}|Q|^{\beta_{2} / n} \\
& \times\left(\int_{0}^{\infty}\left|\int_{\left(\mathbb{R}^{n}\right)^{m}}\left(b_{1}\left(y_{1}\right)-\lambda_{1}\right) K_{t}\left(x, y_{1}, y_{2}\right) f_{1}^{\infty}\left(y_{1}\right) f_{2}^{0}\left(y_{2}\right) d y_{1} d y_{2}\right|^{2} \frac{d t}{t}\right)^{\frac{1}{2}} \\
& +\left\|b_{2}\right\|_{\dot{\lambda}_{\beta_{2}}}|Q|^{\beta_{2} / n} \\
& \times\left(\int_{0}^{\infty}\left|\int_{\left(\mathbb{R}^{n)^{m}}\right.}\left(b_{1}\left(y_{1}\right)-\lambda_{1}\right) K_{t}\left(x, y_{1}, y_{2}\right) f_{1}^{0}\left(y_{1}\right) f_{2}^{\infty}\left(y_{2}\right) d y_{1} d y_{2}\right|^{2} \frac{d t}{t}\right)^{\frac{1}{2}} \\
& +\left\|b_{2}\right\|_{\dot{\lambda}_{\beta_{2}}}|Q|^{\beta_{2} / n} \\
& \times\left(\int_{0}^{\infty}\left|\int_{\left(\mathbb{R}^{n)^{m}}\right.}\left(b_{1}\left(y_{1}\right)-\lambda_{1}\right) K_{t}\left(x, y_{1}, y_{2}\right) f_{1}^{\infty}\left(y_{1}\right) f_{2}^{\infty}\left(y_{2}\right) d y_{1} d y_{2}\right|^{2} \frac{d t}{t}\right)^{\frac{1}{2}} .
\end{aligned}
$$

Then

$$
\begin{aligned}
N_{2} \leq & \left.\frac{C}{|Q|^{1+\beta / n}} \int_{Q}\left|\left\|b_{2}\right\|_{\dot{\lambda}_{\beta_{2}}}\right| Q\right|^{\beta_{2} / n}\left[b_{1}, T\right]\left(f_{1}, f_{2}\right)(z)-c_{1}^{\prime} \mid d z \\
\leq & \frac{C\left\|b_{2}\right\|{\dot{\lambda_{\beta_{2}}}}_{\beta_{2}}}{|Q|^{1+\beta_{1} / n}} \int_{Q}\left|\left(b_{1}(z)-\lambda_{1}\right)\right| T\left(f_{1}, f_{2}\right)(z) d z \\
& +\frac{C\left\|b_{2}\right\| \|_{\dot{\beta}_{2}}}{|Q|^{1+\beta_{1} / n}} \int_{Q} T\left(f_{1}^{0}, f_{2}^{0}\right)(z) d z \\
& +\frac{C\left\|b_{2}\right\|{\dot{\lambda_{\beta}}}_{\beta_{2}}}{|Q|^{1+\beta_{1} / n}} \int_{Q}\left(\int_{0}^{\infty} \mid \int_{\left(\mathbb{R}^{n}\right)^{m}}\left(b_{1}\left(y_{1}\right)-\lambda_{1}\right)\right. \\
& \left.\times\left.\left[K_{t}\left(z, y_{1}, y_{2}\right)-K_{t}\left(x_{Q}, y_{1}, y_{2}\right)\right] f_{1}^{0}\left(y_{1}\right) f_{2}^{\infty}\left(y_{2}\right) d y_{1} d y_{2}\right|^{2} \frac{d t}{t}\right)^{\frac{1}{2}} d z \\
& +\frac{C\left\|b_{2}\right\| \dot{\lambda}_{\beta_{2}}}{|Q|^{1+\beta_{1} / n}} \int_{Q}\left(\int_{0}^{\infty} \mid \int_{\left(\mathbb{R}^{n}\right)^{m}}\left(b_{1}\left(y_{1}\right)-\lambda_{1}\right)\right.
\end{aligned}
$$




$$
\begin{aligned}
& \left.\times\left.\left[K_{t}\left(z, y_{1}, y_{2}\right)-K_{t}\left(x_{Q}, y_{1}, y_{2}\right)\right] f_{1}^{\infty}\left(y_{1}\right) f_{2}^{0}\left(y_{2}\right) d y_{1} d y_{2}\right|^{2} \frac{d t}{t}\right)^{\frac{1}{2}} d z \\
& +\frac{C\left\|b_{2}\right\|{\dot{\lambda_{\beta}}}_{\beta_{2}}}{|Q|^{1+\beta_{1} / n}} \int_{Q}\left(\int_{0}^{\infty} \mid \int_{\left(\mathbb{R}^{n)^{m}}\right.}\left(b_{1}\left(y_{1}\right)-\lambda_{1}\right)\right. \\
& \left.\times\left.\left[K_{t}\left(z, y_{1}, y_{2}\right)-K_{t}\left(x_{Q}, y_{1}, y_{2}\right)\right] f_{1}^{\infty}\left(y_{1}\right) f_{2}^{\infty}\left(y_{2}\right) d y_{1} d y_{2}\right|^{2} \frac{d t}{t}\right)^{\frac{1}{2}} d z \\
\doteq & N_{21}+N_{22}+N_{23}+N_{24}+N_{25} .
\end{aligned}
$$

By the Hölder inequality we have

$$
\begin{aligned}
N_{21} & \leq C\left\|b_{2}\right\|_{\dot{\beta}_{\beta_{2}}}\left(\frac{1}{|Q|^{r^{\prime} \beta_{1} / n+1}} \int_{Q}\left|b_{1}(z)-\lambda_{1}\right|^{r^{\prime}} d z\right)^{1 / r^{\prime}}\left(\frac{1}{|Q|} \int_{Q}\left|T\left(f_{1}, f_{2}\right)(z)\right|^{r} d z\right)^{1 / r} \\
& \leq\left\|b_{1}\right\|_{{\dot{\beta_{1}}}_{1}}\left\|b_{2}\right\|_{{\dot{\beta_{2}}}_{2} M_{r}\left(T\left(f_{1}, f_{2}\right)\right)(x) .}
\end{aligned}
$$

Take $1<q_{1}<p_{1}, 1<q_{2}<p_{2}$, and $1<q<\infty$ such that $1 / q=1 / q_{1}+1 / q_{2}$. Then by the Hölder inequality and Lemma 2.3 we have

$$
\begin{aligned}
N_{22} & \leq \frac{C\left\|b_{2}\right\|_{{\dot{\beta}}_{2}}}{|Q|^{\beta_{1} / n+1 / q}}\left(\int_{Q}\left|T\left(\left(b_{1}-\lambda_{1}\right) f_{1}^{0}, f_{2}^{0}\right)(z)\right|^{q} d z\right)^{1 / q} \\
& \leq \frac{C\left\|b_{2}\right\|_{\dot{\lambda}_{\beta_{2}}}}{|Q|^{\beta_{1} / n+1 / q}}\left\|\left(b_{1}-\lambda_{1}\right) f_{1}^{0}\right\|_{L^{q_{1}}}\left\|f_{2}^{0}\right\|_{L^{q_{2}}} \\
& \leq \frac{C\left\|b_{1}\right\|{\dot{\lambda}_{\beta_{1}}}\left\|b_{2}\right\|_{\dot{\lambda}_{\beta_{2}}}}{|Q|^{1 / q}}\left\|f_{1}^{0}\right\|_{L^{q_{1}}}\left\|f_{2}^{0}\right\|_{L^{q_{2}}} \\
& \leq\left\|b_{1}\right\|_{{\dot{\beta_{1}}}_{1}}\left\|b_{2}\right\|_{{\dot{\beta_{2}}}_{2}} M_{q_{1}}\left(f_{1}\right)(x) M_{q_{2}}\left(f_{2}\right)(x) .
\end{aligned}
$$

For $y_{2} \in\left(Q^{*}\right)^{c},\left|y_{2}-x_{Q}\right| \sim\left|y_{2}-z\right|$, and $\left|z-x_{Q}\right| \leq \frac{\left|y_{2}-z\right|}{2} \leq \frac{1}{2} \max \left\{\left|z-y_{1}\right|,\left|z-y_{2}\right|\right\}$, by Minkowski's inequality and the smooth condition (1.2) we get

$$
\begin{aligned}
N_{23} \leq & \frac{C\left\|b_{2}\right\|_{\dot{\lambda}_{\beta_{2}}}}{|Q|^{1+\beta_{1} / n}} \int_{Q} \int_{\left(\mathbb{R}^{n}\right)^{2}}\left|\left(b_{1}\left(y_{1}\right)-\lambda_{1}\right)\right| \\
& \times\left(\int_{0}^{\infty}\left|K_{t}\left(z, y_{1}, y_{2}\right)-K_{t}\left(x_{Q}, y_{1}, y_{2}\right)\right|^{2} \frac{d t}{t}\right)^{\frac{1}{2}}\left|f_{1}^{0}\left(y_{1}\right) f_{2}^{\infty}\left(y_{2}\right)\right| d y_{1} d y_{2} d z \\
\leq & \frac{C\left\|b_{1}\right\|_{\dot{\lambda}_{\beta_{1}}}\left\|b_{2}\right\|_{\dot{\lambda}_{\beta_{2}}}}{|Q|^{1+\beta_{1} / n}} \int_{Q} \int_{\left(\mathbb{R}^{n}\right)^{2}} \frac{\left|y_{1}-x_{Q}\right|^{\beta_{1}}}{\left(\left|z-y_{1}\right|+\left|z-y_{2}\right|\right)^{2 n}} \\
& \times \omega\left(\frac{\left|z-x_{Q}\right|}{\left|z-y_{1}\right|+\left|z-y_{2}\right|}\right)\left|f_{1}^{0}\left(y_{1}\right) f_{2}^{\infty}\left(y_{2}\right)\right| d y_{1} d y_{2} d z \\
\leq & \frac{C\left\|b_{1}\right\|{\dot{\lambda_{\beta}}}_{\beta_{1}}\left\|b_{2}\right\|{\dot{\lambda_{\beta_{2}}}}_{\beta_{2}}}{|Q|^{1+\beta_{1} / n}} \int_{Q} \frac{\left|f_{1}^{0}\left(y_{1}\right) f_{2}^{\infty}\left(y_{2}\right)\right|}{\left.\left|z-y_{1}\right|+\left|z-y_{2}\right|\right)^{2 n-\beta_{1}}} \\
& \times \omega\left(\frac{\left|z-x_{Q}\right|}{\left|z-y_{1}\right|+\left|z-y_{2}\right|}\right) d y_{1} d y_{2} d z \\
\leq & \frac{C}{|Q|}\left\|b_{1}\right\|_{\dot{\lambda}_{\beta_{1}}}\left\|b_{2}\right\|_{\dot{\lambda}_{\beta_{2}}} \int_{Q} \int_{Q^{*}}\left|f_{1}\left(y_{1}\right)\right| \int_{\left(Q^{*}\right)^{c}} \frac{\left|f_{2}\left(y_{2}\right)\right|}{\left|z-y_{2}\right|^{2 n}} \omega\left(\frac{\left|z-x_{Q}\right|}{\left|z-y_{2}\right|}\right) d y_{2} d y_{1} d z
\end{aligned}
$$




$$
\begin{aligned}
& \leq \frac{C}{|Q|}\left\|b_{1}\right\|_{{\dot{\beta_{1}}}_{1}}\left\|b_{2}\right\|_{{\dot{\beta_{2}}}_{2}} \int_{Q} \int_{Q^{*}}\left|f_{1}\left(y_{1}\right)\right| \\
& \times \sum_{k=1}^{\infty} \int_{2^{k+3} \sqrt{n} Q \backslash 2^{k+2} \sqrt{n} Q}\left|f_{2}\left(y_{2}\right)\right|\left|2^{k} \sqrt{n} Q\right|^{-2} \omega\left(2^{-k}\right) d y_{2} d y_{1} d z \\
& \leq C\left\|b_{1}\right\|{\dot{\lambda}_{\beta_{1}}}_{\| b_{2}} \|_{{\dot{\beta_{2}}}_{\beta_{2}}} \frac{1}{|Q|} \int_{Q^{*}}\left|f_{1}\left(y_{1}\right)\right| d y_{1} \\
& \times \sum_{k=1}^{\infty}|Q|\left|2^{k+3} \sqrt{n} Q\right|^{-1} \omega\left(2^{-k}\right) \frac{1}{\left|2^{k+3} \sqrt{n} Q\right|} \int_{2^{k+3} \sqrt{n} Q \backslash 2^{k+2} \sqrt{n} Q}\left|f_{2}\left(y_{2}\right)\right| d y_{2} \\
& \leq C\left\|b_{1}\right\|_{{\dot{\beta_{1}}}_{1}}\left\|b_{2}\right\|_{{\dot{\beta_{2}}}_{2}} M\left(f_{1}\right)(x) \sum_{k=1}^{\infty} 2^{-k} \omega\left(2^{-k}\right) \frac{1}{\left|2^{k+3} \sqrt{n} Q\right|} \\
& \times \int_{2^{k+3} \sqrt{n} Q \backslash 2^{k+2} \sqrt{n} Q}\left|f_{2}\left(y_{2}\right)\right| d y_{2} \\
& \leq C\left\|b_{1}\right\|_{{\dot{\beta_{1}}}_{1}}\left\|b_{2}\right\|_{{\dot{\beta_{2}}}_{2}} M\left(f_{1}\right)(x) M\left(f_{2}\right)(x) .
\end{aligned}
$$

Similarly,

$$
N_{24} \leq C\left\|b_{1}\right\|_{{\dot{\beta_{1}}}_{1}}\left\|b_{2}\right\|_{{\dot{\beta_{2}}}_{2}} M\left(f_{1}\right)(x) M\left(f_{2}\right)(x)
$$

For $y_{1}, y_{2} \in\left(Q^{*}\right)^{c},\left|y_{1}-x_{Q}\right| \sim\left|y_{1}-z\right|$, and $\left|y_{2}-x_{Q}\right| \sim\left|y_{2}-z\right|$, by Minkowski's inequality and the smooth condition (1.2) we get

$$
\begin{aligned}
& N_{25} \leq \frac{C\left\|b_{2}\right\|_{\dot{\lambda}_{\beta_{2}}}}{|Q|^{1+\beta_{1} / n}} \int_{Q} \int_{\left.\mathbb{R}^{n}\right)^{2}}\left|\left(b_{1}\left(y_{1}\right)-\lambda_{1}\right)\right| \\
& \times\left(\int_{0}^{\infty}\left|K_{t}\left(z, y_{1}, y_{2}\right)-K_{t}\left(x_{Q}, y_{1}, y_{2}\right)\right|^{2} \frac{d t}{t}\right)^{\frac{1}{2}}\left|f_{1}^{\infty}\left(y_{1}\right) f_{2}^{\infty}\left(y_{2}\right)\right| d y_{1} d y_{2} d z \\
& \leq \frac{C\left\|b_{1}\right\|{\dot{\lambda_{\beta}}}_{\beta_{1}}\left\|b_{2}\right\|_{{\dot{\lambda_{\beta}}}_{2}}}{|Q|^{1+\beta_{1} / n}} \int_{Q} \int_{\left(\mathbb{R}^{n}\right)^{2}} \frac{\left|y_{1}-x_{Q}\right|^{\beta_{1}}}{\left(\left|z-y_{1}\right|+\left|z-y_{2}\right|\right)^{2 n}} \\
& \times \omega\left(\frac{\left|z-x_{Q}\right|}{\left|z-y_{1}\right|+\left|z-y_{2}\right|}\right)\left|f_{1}^{\infty}\left(y_{1}\right) f_{2}^{\infty}\left(y_{2}\right)\right| d y_{1} d y_{2} d z \\
& \leq \frac{C\left\|b_{1}\right\|{\dot{i_{\beta}}}_{\beta_{1}}\left\|b_{2}\right\|{\dot{\lambda_{\beta_{2}}}}_{\left.\right|^{1}}}{|Q|^{1+\beta_{1} / n}} \int_{Q} \int_{\left(\mathbb{R}^{n}\right)^{2}} \frac{\left|y_{1}-x_{Q}\right|^{\beta_{1}}\left|f_{1}^{0}\left(y_{1}\right) f_{2}^{\infty}\left(y_{2}\right)\right|}{\left(\left|z-y_{1}\right|+\left|z-y_{2}\right|\right)^{2 n}} \\
& \times \omega\left(\frac{\left|z-x_{Q}\right|}{\left|z-y_{1}\right|+\left|z-y_{2}\right|}\right) d y_{1} d y_{2} d z \\
& \leq \frac{C}{|Q|^{1+\beta_{1} / n}}\left\|b_{1}\right\|_{{\dot{\beta_{1}}}_{1}}\left\|b_{2}\right\|_{{\dot{\beta_{2}}}_{\beta_{2}}} \int_{Q} \int_{\left(\left(Q^{*}\right)^{c}\right)^{2}} \frac{\left|f_{1}\left(y_{1}\right)\right|\left|f_{2}\left(y_{2}\right)\right|}{\left|y_{1}-x_{Q}\right|^{2 n-\beta_{1}}} \omega\left(\frac{\left|z-x_{Q}\right|}{\left|z-y_{1}\right|}\right) d y_{1} d y_{2} d z \\
& \leq \frac{C}{|Q|^{1+\beta_{1} / n}}\left\|b_{1}\right\|{\dot{\lambda}_{\beta_{1}}}\left\|b_{2}\right\|_{{\dot{\beta_{2}}}_{\beta_{2}}} \\
& \times \int_{Q} \sum_{k=1}^{\infty} \int_{2^{k+3} \sqrt{n} Q 22^{k+2} \sqrt{n} Q} \frac{\left|f_{1}\left(y_{1}\right)\right|\left|f_{2}\left(y_{2}\right)\right|}{\left|y_{1}-x_{Q}\right|^{2 n-\beta_{1}}} \omega\left(\frac{\left|z-x_{Q}\right|}{\left|z-y_{1}\right|}\right) d y_{1} d y_{2} d z \\
& \leq C\left\|b_{1}\right\|{\dot{\lambda_{\beta}}}_{\beta_{1}}\left\|b_{2}\right\|_{{\dot{\beta_{2}}}_{2}} \sum_{k=1}^{\infty} \frac{2^{k \beta_{1}} \omega\left(2^{-k}\right)}{\left|2^{k+3} \sqrt{n} Q\right|^{2}}
\end{aligned}
$$




$$
\begin{aligned}
& \times \int_{2^{k+3} \sqrt{n} Q \backslash 2^{k+2} \sqrt{n} Q}\left|f_{1}\left(y_{1}\right)\right| d y_{1} \int_{2^{k+3} \sqrt{n} Q \backslash 2^{k+2} \sqrt{n} Q}\left|f_{2}\left(y_{2}\right)\right| d y_{2} \\
\leq & C\left\|b_{1}\right\|_{\dot{\lambda}_{\beta_{1}}}\left\|b_{2}\right\|_{{\dot{\beta_{2}}}_{2}} M\left(f_{1}\right)(x) M\left(f_{2}\right)(x) .
\end{aligned}
$$

Combining the estimates for $N_{21}, N_{22}, N_{23}, N_{24}, N_{25}$, we get

$$
N_{2} \leq C\left\|b_{1}\right\|_{{\dot{\beta_{1}}}_{1}}\left\|b_{2}\right\|_{{\dot{\beta_{2}}}_{2}}\left\{M_{r}\left(T\left(f_{1}, f_{2}\right)\right)(x)+M_{q_{1}}\left(f_{1}\right)(x) M_{q_{2}}\left(f_{2}\right)(x)+M\left(f_{1}\right)(x) M\left(f_{2}\right)(x)\right\} .
$$

Similarly, we have

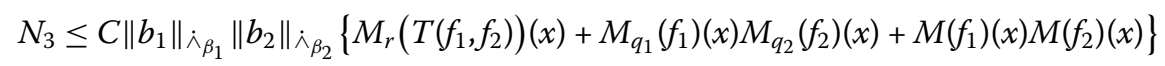

and

$$
N_{4} \leq C\left\|b_{1}\right\|_{i_{\beta_{1}}}\left\|b_{2}\right\|_{i_{\beta_{2}}}\left\{M_{r}\left(T\left(f_{1}, f_{2}\right)\right)(x)+M_{q_{1}}\left(f_{1}\right)(x) M_{q_{2}}\left(f_{2}\right)(x)+M\left(f_{1}\right)(x) M\left(f_{2}\right)(x)\right\} .
$$

Thus we deduce that

$$
\begin{aligned}
& \frac{1}{|Q|^{1+\beta / n}} \int_{Q}\left|T_{\Pi \vec{b}}(\vec{f})(z)-\left(T_{\Pi \vec{b}}(\vec{f})\right)_{Q}\right| d z \\
& \quad \leq C\left\|b_{1}\right\|_{\dot{\lambda}_{\beta_{1}}}\left\|b_{2}\right\|_{\dot{\lambda}_{\beta_{2}}}\left\{M_{r}\left(T\left(f_{1}, f_{2}\right)\right)(x)+M_{q_{1}}\left(f_{1}\right)(x) M_{q_{2}}\left(f_{2}\right)(x)+M\left(f_{1}\right)(x) M\left(f_{2}\right)(x)\right\} .
\end{aligned}
$$

By the Hölder inequality, Lemma 2.3, and the normal inequalities for the maximal operators, we arrive at

$$
\begin{aligned}
& \left\|T_{\Pi \vec{b}}(\vec{f})\right\|_{\dot{F}_{p}^{\beta, \infty}} \\
& \approx\left\|\sup _{Q} \frac{1}{|Q|^{1+\beta / n}} \int_{Q}\left|T_{\Pi \vec{b}}(\vec{f})(z)-\left(T_{\Pi \vec{b}}(\vec{f})\right)_{Q}\right| d z\right\|_{L^{p}} \\
& \leq C\left\|b_{1}\right\|_{{\dot{\beta_{1}}}_{1}}\left\|b_{2}\right\|_{{\dot{\beta_{2}}}_{2}}\left\{\left\|M_{r}\left(T\left(f_{1}, f_{2}\right)\right)\right\|_{L^{p}}+\left\|M_{q_{1}}\left(f_{1}\right) M_{q_{2}}\left(f_{2}\right)\right\|_{L^{p}}+\left\|M\left(f_{1}\right) M\left(f_{2}\right)\right\|_{L^{p}}\right\} \\
& \leq C\left\|b_{1}\right\|_{{\dot{\beta_{1}}}_{1}}\left\|b_{2}\right\|_{{\dot{\lambda_{2}}}_{2}}\left\{\left\|T\left(f_{1}, f_{2}\right)\right\|_{L^{p}}+\left\|M_{q_{1}}\left(f_{1}\right)\right\|_{L^{p_{1}}}\left\|M_{q_{2}}\left(f_{2}\right)\right\|_{L^{p_{2}}}\right. \\
& \left.+\left\|M\left(f_{1}\right)\right\|_{L^{p_{1}}}\left\|M\left(f_{2}\right)\right\|_{L^{p_{2}}}\right\} \\
& \leq\left\|b_{1}\right\|_{{\dot{\beta_{1}}}_{1}}\left\|b_{2}\right\|_{{\dot{\beta_{2}}}_{2}}\left\|f_{1}\right\|_{L^{p_{1}}}\left\|f_{2}\right\|_{L^{p_{2}}}
\end{aligned}
$$

where we have used that facts $1<r<p, 1<q_{1}<p_{1}$, and $1<q_{2}<p_{2}$. This finishes the proof of Theorem 1.3.

\section{Conclusions}

In this paper,we studied the boundedness properties of the commutator generated by a multilinear square function and Lipschitz functions with kernel satisfying Dini-type condition. We showed that such commutators are bounded from product Lebesgue spaces into the Lebesgue spaces, Lipschitz spaces, and Triebel-Lizorkin spaces. 


\section{Funding}

The first author was supported partly by the Key Research Project for Higher Education in Henan Province

(No. 19A110017). The second author was supported partly by NSFC (Nos. 11471041, 11671039) and NSFC-DFG (No.

11761131002).

\section{Competing interests}

The authors declare that they have no competing interests.

\section{Authors' contributions}

Both authors read and approved the final manuscript.

\section{Author details}

${ }^{1}$ School of Mathematics and Information Science, Henan Polytechnic University, Jiaozuo, People's Republic of China. ${ }^{2}$ School of Mathematical Sciences, Beijing Normal University, Beijing, People's Republic of China. ${ }^{3}$ Laboratory of Mathematics and Complex Systems, Ministry of Education, Beijing, People's Republic of China.

\section{Publisher's Note}

Springer Nature remains neutral with regard to jurisdictional claims in published maps and institutional affiliations.

Received: 26 March 2018 Accepted: 17 July 2018 Published online: 25 July 2018

\section{References}

1. Chanillo, S.: A note on commutators. Indiana Univ. Math. J. 31, 7-16 (1982)

2. Chen, X., Xue, Q., Yabuta, K.: On multilinear Littlewood-Paley operators. Nonlinear Anal. 115, 25-40 (2015)

3. Coifman, R.R., Deng, D., Meyer, Y.: Domains de la racine carrée de certains opérateurs différentiels accrétifs. Ann. Inst. Fourier (Grenoble) 33, 123-134 (1983)

4. David, G., Journe, J.L.: Une caractérisation des opérateurs intégraux singuliers bornés sur $L^{2}\left(\mathbb{R}^{n}\right)$. C. R. Math. Acad. Sci. Paris 296, 761-764 (1983)

5. Fabes, E.B., Jerison, D., Kenig, C.: Multilinear Littlewood-Paley estimates with applications to partial differential equations. Proc. Natl. Acad. Sci. 79, 5746-5750 (1982)

6. Fabes, E.B., Jerison, D., Kenig, C.: Multilinear square functions and partial differential equations. Am. J. Math. 107, 1325-1368 (1985)

7. Hart, J.: Bilinear square functions and vector-valued Calderón-Zygmund operators. J. Fourier Anal. Appl. 18, 1291-1313 (2012)

8. Jie, S., Zhang, P.: Commutators of multilinear Calderón-Zygmund operators with Dini type kernels on some function spaces. J. Nonlinear Sci. Appl. 10, 5002-5019 (2017)

9. Lu, G., Zhang, P: Multilinear Calderón-Zygmund operators with kernels of Dini's type and applications. Nonlinear Anal. 107, 92-117 (2014)

10. Maldonado, D., Naibo, V.: Weighted norm inequalities for paraproducts and bilinear pseudodifferential operators with mild regularity. J. Fourier Anal. Appl. 15, 218-261 (2009)

11. Mo, H., Lu, S.: Commutators generated by multilinear Calderón-Zygmund type singular integral and Lipschitz functions. Acta Math. Appl. Sin. 40(3), 903-912 (2014)

12. Paluszynski, M.: Characterization of the Besov spaces via the commutator operator of Coifman, Rochberg and Weiss. Indiana Univ. Math. J. 44, 1-18 (1995)

13. Sato, S., Yabuta, K.: Multilinearized Littlewood-Paley operators. Sci. Math. Jpn. 55(3), 447-453 (2002)

14. Shi, S., Xue, Q., Yabuta, K.: On the boundedness of multilinear Littlewood-Paley $g_{\lambda}^{*}$ function. J. Math. Pures Appl. 101(3), 394-413 (2014)

15. Si, Z., Xue, Q: Multilinear square functions with kernels of Dini's type. J. Funct. Spaces 2016, Article ID 4876146 (2016)

16. Wang, $\mathrm{W} . \mathrm{Xu}, \mathrm{J}$.: Commutators of multilinear singular integrals with Lipschitz functions. Commun. Math. Res. 25(4), 318-328 (2009)

17. Xue, Q., Peng, X., Yabuta, K.: On the theory of multilinear Littlewood-Paley g function. J. Math. Soc. Jpn. 67(2), 535-559 (2015)

18. Xue, Q., Yan, J.: On multilinear square function and its applications to multilinear Littlewood-Paley operators with non-convolution type kernels. J. Math. Anal. Appl. 422, 1342-1362 (2015)

19. Yabuta, K.: A multilinearization of Littlewood-Paley's g-function and Carleson measures. Tohoku Math. J. 34, 251-275 (1982) 